\title{
TOWARDS IMPROVED ESTIMATES OF UPPER OCEAN ENERGETICS
}

\author{
A Thesis \\ presented to \\ the Faculty of California Polytechnic State University, \\ San Luis Obispo
}

\author{
In Partial Fulfillment \\ of the Requirements for the Degree \\ Master of Science in Electrical Engineering
}

by

Alexander Grant Wineteer

March 2016 
(C) 2016

Alexander Grant Wineteer

ALL RIGHTS RESERVED 


\section{COMMITTEE MEMBERSHIP}

TITLE: Towards Improved Estimates of Upper Ocean Energetics

AUTHOR: Alexander Grant Wineteer

DATE SUBMITTED: March 2016

COMMITTEE CHAIR: John Saghri, Ph.D.

Professor of Electrical Engineering

COMMITTEE MEMBER: Jane Zhang, Ph.D.

Professor of Electrical Engineering

COMMITTEE MEMBER: Ryan Walter, Ph.D.

Assistant Professor of Physics 


\section{ABSTRACT \\ Towards Improved Estimates of Upper Ocean Energetics}

Alexander Grant Wineteer

The energy exchanged between the atmosphere and the ocean is an important parameter in understanding the Earth's climate. One way of quantifying this energy exchange is through the use of "wind work," or the work done on the ocean by the wind. Since wind work is calculated according to the interaction between ocean surface currents and surface wind stress, a number of surface current decompositions can be used to decompose wind work calculations. In this research, geostrophic, ageostrophic, Ekman, and total current decompositions are all used to calculate wind work. Geostrophic currents are formed by the balance of surface pressure gradients and the Coriolis effect. Ageostrophic currents, on the other hand, are difficult to calculate because they are made up of many types of currents, and are generally defined as any current not in geostrophic balance. The main component of ageostrophic currents, Ekman currents, are used in this work to approximate ageostrophic currents. Ekman currents are formed by the balance of surface wind stress and the Coriolis effect. Finally, total currents are the sum of all currents in the ocean.

Using high resolution, global NASA ocean models, the wind work on the global oceans is estimated via a number of decompositions, with results finding about $3.2 \mathrm{TW}, .32 \mathrm{TW}$, and 3.05 TW for total, geostrophic, and Ekman wind work respectively, when taking a 7 day window average of surface currents and a 1 day average of surface stress. Averaging period for currents is found to significantly affect the resulting calculated wind work, with greater than 50 percent difference between 1 and 15 days of averaging. Looking at the same total, geostrophic, and Ekman wind work results for 1 day averages of wind stress and surface currents finds $5.5 \mathrm{TW}, .03 \mathrm{TW}$, and $6.3 \mathrm{TW}$ respectively. This result indicates that high frequency currents are very important to wind work. Seasonally, wind work is found to be at a maximum during the Northern Hemisphere $(\mathrm{NH})$ summer, and at a minimum during the NH winter months.

To help motivate the funding of a Doppler Scatterometer, simulations are used to show the capabilities of such an instrument in measuring wind work. DopplerScat simulations find that a satellite capable of measuring coincident surface vector winds and surface vector currents, with $1.1 \mathrm{~m} / \mathrm{s}$ wind speed error and $.5 \mathrm{~m} / \mathrm{s}$ current speed error, could estimate global wind work to within 2 percent accuracy on an 8 day average with daily global snapshots. 


\section{ACKNOWLEDGMENTS}

To all the friends, family, mentors, and teachers that have supported me in my life:

I am truly fortunate to be surrounded by such amazing people.

Thank you.

This work was carried out at Cal Poly, San Luis Obispo, under contract with Caltech and the National Aeronautics and Space Administration. Government sponsorship acknowledged. 


\section{TABLE OF CONTENTS}

LIST OF TABLES

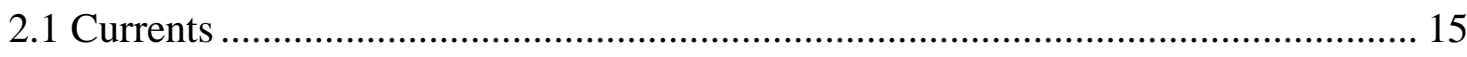

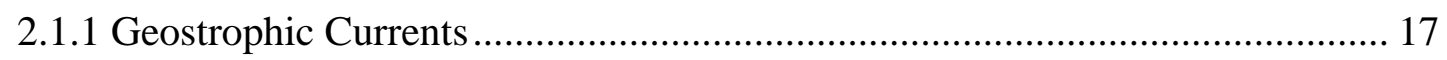

2.1.2 Ageostrophic Currents .............................................................................. 19

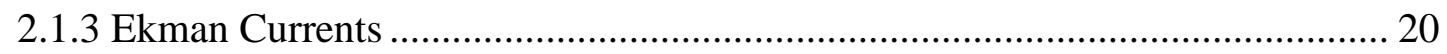

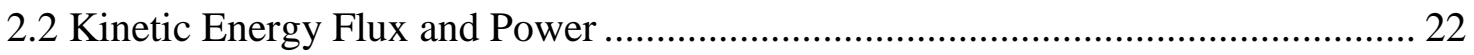

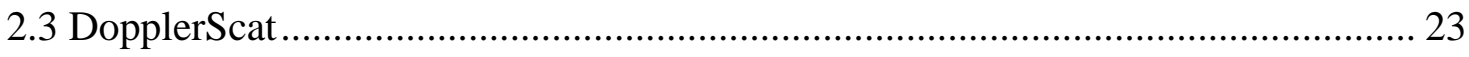

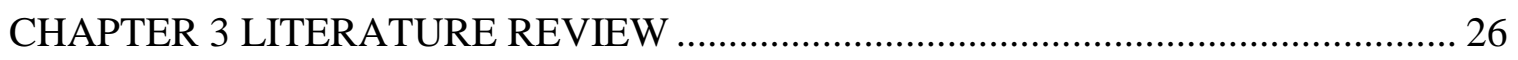

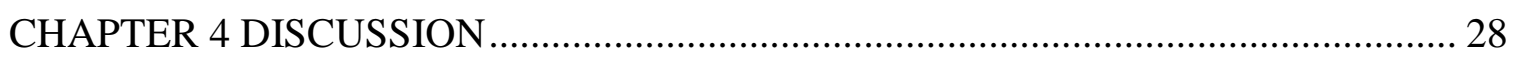

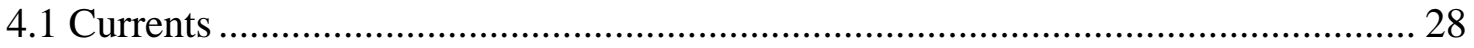

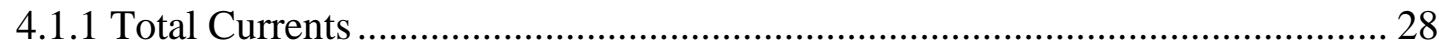

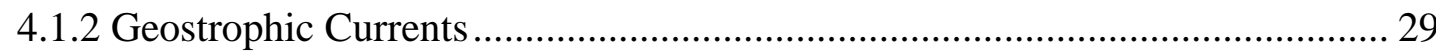

4.1.3 Ekman Currents ................................................................................... 31

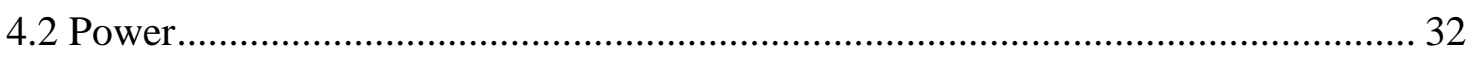

4.2.1 Total (MITgcm) Power.............................................................................. 32

4.2.2 Geostrophic Power ……………………………........................................ 34

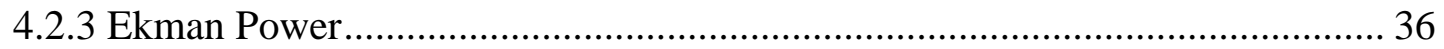

4.2.4 Ageostrophic Power ………………………………................................. 37

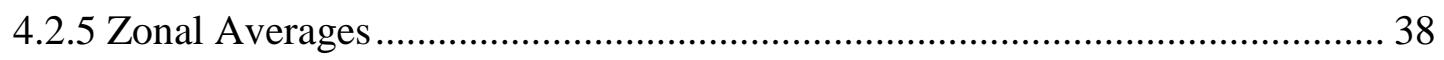

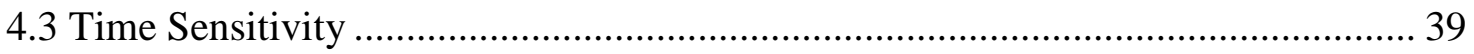

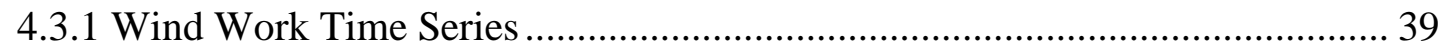

4.3.2 Averaging Period .................................................................................... 42

4.4 Spatial Resolution Effects .......................................................................... 46

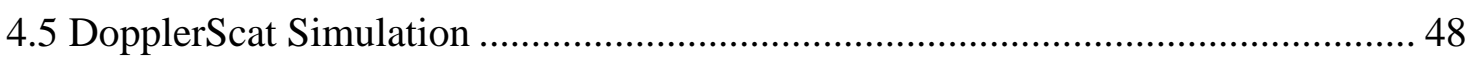




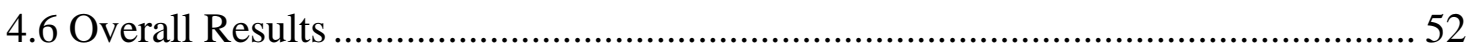

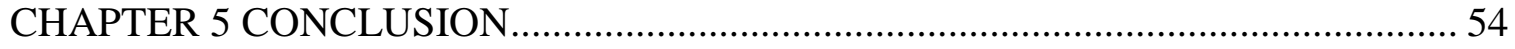

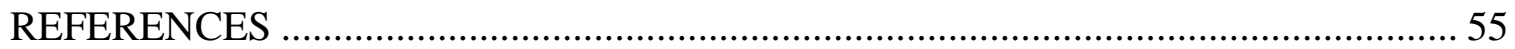




\section{LIST OF TABLES}

Table

Page

Table 1: Variable statistics for Sea Surface Height. ..................................................... 5

Table 2: Model variables used for computations in this work.................................. 6

Table 3: Filter coefficients for 24 sample Hamming filter. ....................................... 8

Table 4: Wind work results for each current type and averaging period. Units in TW... 53 


\section{LIST OF FIGURES}

Figure

Page

Figure 1: LLC grid layout. Faces labeled with numbers 1-5; the smaller cells represent

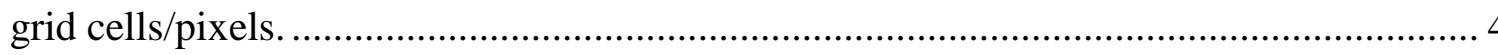

Figure 2: Latitude of grid cells in LLC-4320 1/48 degree model................................... 6

Figure 3: Magnitude response for 24 sample Hamming filter..................................... 7

Figure 4: Phase response of 24 sample Hamming filter. .......................................... 8

Figure 5: Original Sea Surface Height map with ice appearing in red. .......................... 11

Figure 6: Sea Surface Height after first pass of ice removal. ..................................... 11

Figure 7: Final Sea Surface Height after filtering....................................................... 12

Figure 8: Process diagram of work done in this research. ......................................... 15

Figure 9: Rossby number for $1 / 48$ degree model..................................................... 19

Figure 10: Optimization for vertical viscosity results in Az of .014 ........................... 21

Figure 11: Comparison of Parzen and Gaussian filters of length 4............................... 24

Figure 12: Total currents as output by the MITgcm model. Seven day average during

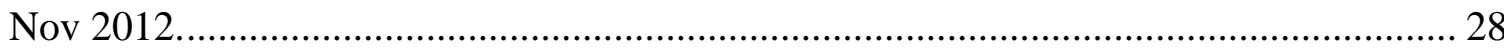

Figure 13: Geostrophic currents as calculated based on a seven day average of Sea

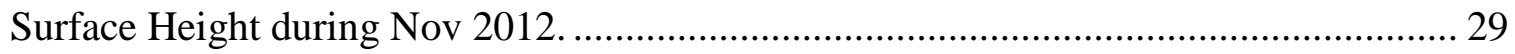

Figure 14: Geostrophic currents plotted over sea surface height in the Southern Ocean off the tip of Southern Africa. Seven day averages shown......................................... 30

Figure 15: Geostrophic vectors plotted over 3D sea surface height data. Again, off the

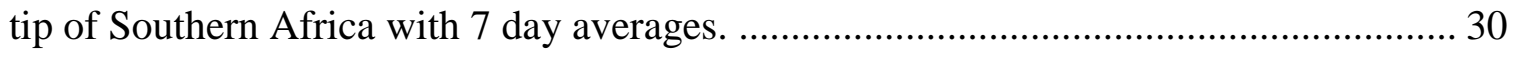


Figure 16: Ekman currents as calculated based on a seven day average of surface stress

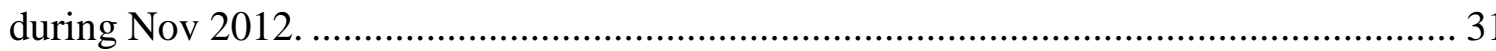

Figure 17: Left to right: Total model surface currents (7d avg), surface stress (1d avg), and KE flux computed from the previous two. Single average from Nov 2012. 32 Figure 18: Zoom of Figure 17 for the Gulf Stream. Total surface currents, surface stress, and KE flux from left to right. 33 Figure 19: Left to right: Geostrophic surface currents computed from a 7d avg of sea surface height, 1d avg of surface stress, and KE flux computed from the previous two.

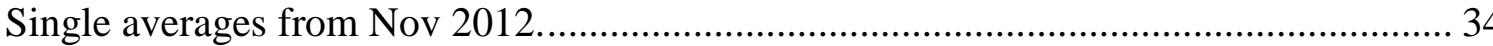

Figure 20: Zoom plot of Figure 19 for the Gulf Stream. Geostrophic currents, surface stress, and KE flux computed from the previous two from left to right 35 Figure 21: Surface Ekman currents, surface stress, and kinetic energy flux computed from the previous two. 36 Figure 22: Zoom plot of Figure 21 for the Gulf Stream. From left to right: Ekman currents, surface stress, and KE flux computed from the previous two.

Figure 23: Comparison of KE flux for Ekman currents (left) and Ageostrophic currents (right).

Figure 24: Zonal averages of kinetic energy flux for total model, Ekman, geostrophic, and the total approximation geostrophic+Ekman.

Figure 25: Time series for total power over 15 months on $1 / 24^{\text {th }}$ degree data. Seven day window averaging used for currents, one day for surface stress. 41 
Figure 26: Seasonal averages for one year of $1 / 24^{\text {th }}$ degree data. Seven day window averaging for currents, one day for surface stress. Standard error bars are optimistic due to autocorrelation.

Figure 27: The effect of averaging period on wind work. Data taken from $1 / 48^{\text {th }}$

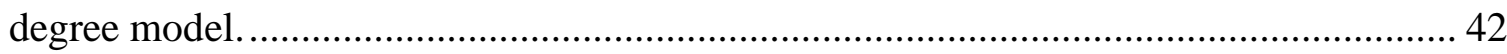

Figure 28: Zonal averages of KE flux for total currents over multiple current averaging periods. Surface stress averaging held constant at one day. 44

Figure 29: Zonal averages of KE flux for Ekman currents over multiple current averaging periods. Surface stress averaging held constant at one day .45 Figure 30: Zonal averages of KE flux for Geostrophic currents over multiple current averaging periods. Surface stress averaging held constant at one day. .45

Figure 31: Averaging period and resolution effects on global wind work .46

Figure 32: Spatial maps of $1 / 24^{\text {th }}$ degree total power minus $1 / 48^{\text {th }}$ degree total power for a 7 day average. Both maps are the same, but with different color scales................ 47

Figure 33: No noise, filtered and downsampled total currents KE flux. ....................... 48 Figure 34: Filtered, downsampled, noisy KE flux computed from total currents. ........... 49 Figure 35: Comparison of DopplerScat noisy KE flux to $1 / 48^{\text {th }}$ degree model data....... 50

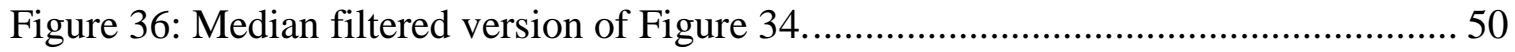
Figure 37: Zonal average comparison of KE flux for noisy and no noise DopplerScat simulations 51 


\section{CHAPTER 1 INTRODUCTION}

Ocean surface energetics are integral to understanding the Earth's climate. Nutrient dispersal, pollutant dispersal, climate change, and heat transport are all significantly and directly affected by the conditions at the surface of the ocean, and arguably, largely by the energy interactions at the surface. In other words, the interaction between the atmosphere and the ocean is important. One way of studying this interaction is through the use of coincident measurements of ocean surface currents and near-surface wind velocity. Taken together, these two velocity measurements can be used to calculate kinetic energy flux through the ocean surface - the amount of energy input into the ocean from wind. This calculation, and variations on it, will be the focus of this work.

While scientifically important, and certainly interesting, the above goals also have some more practical applications. Using expected error budgets, the above power calculations will be transformed into simulated ocean observations to help motivate the funding of a satellite borne instrument capable of measuring the inputs for wind work.

The general goal of this research is to calculate global wind work, which is the globally integrated kinetic energy flux due to wind at the surface of the ocean. Kinetic energy flux is calculated as the dot product of surface stress and surface currents in the ocean. While the calculation itself is fairly straightforward, there are many different decompositions that can be used to calculate different types of currents, and thus, wind work on each type of current. Geostrophic currents, ageostrophic currents, Ekman currents, and total currents are considered in this work.

Total currents are simply the sum of all currents at the surface of the ocean, and are what an observer would see if they looked at the ocean. Geostrophic currents are a 
type of current that is formed by a balance between surface pressure gradients and the Coriolis effect. Since sea level is related to surface pressure, altimeter measured sea surface height can be used to calculate geostrophic currents. Ageostrophic currents, on the other hand, are a group of many different types of currents, but are generally defined as any current not in geostrophic balance. Finally, Ekman currents are the main subgroup of Ageostrophic currents, and are formed by the balance of surface wind stress (the wind blowing across the surface of the ocean) and the Coriolis effect. Since surface wind stress is the driver for Ekman currents, these types of currents may be calculated based solely on wind vectors. Ekman currents are used as an approximation for ageostrophic currents in this research.

In calculating wind work, the choice of surface stress parameterization is also very important. Wind stress is the frictional pressure applied to the surface of the ocean by the wind. Many previous wind work studies have assumed that the wind stress is simply a function of the surface wind speed, but this work will assume that it is the relative velocity of surface winds compared to surface currents that matters.

In summary, to calculate the decompositions of wind work, sea surface height, wind stress, and total currents are necessary. Since global measurements of total surface currents do not yet exist, an ocean model will be used for all calculations in this work. 


\subsection{Ocean Model}

An extension of the ECCO2 (Estimating the Circulation and Climate of the Ocean) model family was used in this work. The model used is a global ocean model driven by the Massachusetts Institute of Technology General Circulation Model $(\mathrm{MITgcm})$, and is capable of resolving internal tides and sub-mesoscale variability at the

$1 / 48^{\text {th }}$ degree resolution used. The model was developed at the Jet Propulsion Laboratory (Menemenlis, personal communication), and is a very high resolution, global scale, physics based model.

Model data output is provided in the form of hourly binary data files. Each data file contains within it a single variable (for example, sea surface height), with data points specified at specific 2-dimensional grid points. Depending on the variable, some data files contain data at 3-dimensional grid points, extending down into the ocean. Unfortunately, data files are not provided in their gridded form, so code had to be written to create gridded, useable data sets for this work. This was a significant effort and there are a number of nuances in the data that made gridding difficult. I hope that this writing will help future researchers in understanding the LLC (lat-lon-cap) high resolution grid system. 
Data is stored in a gridded but rotated form, shown in Figure 1. Each numbered region is referred to as a "face." Faces $1,2,4$, and 5 are each the same size, while face 3 is smaller. Faces 4 and 5 are rotated 90 degrees relative to the compass directions. This rotation causes a number of issues for gridding data, particularly when dealing with

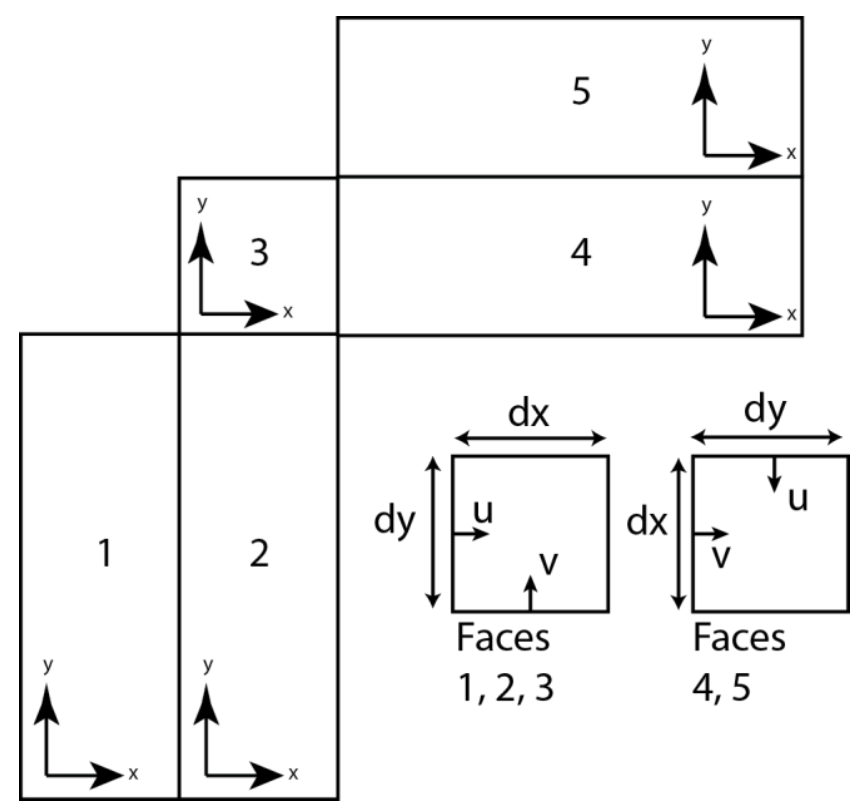

Figure 1: LLC grid layout. Faces labeled with numbers 1-5; the smaller cells represent grid cells/pixels.

vector data. Vector surface currents, for example, are reversed and changed in sign on rotated faces $(U=V, V=-U)$. Since the gridding system does not center vectors on tracer coordinates (grid centers), and instead centers vectors on grid lines, switching $\mathrm{U}$ and $\mathrm{V}$ in faces 4 and 5 causes a shift by one grid cell that must be corrected.

Face 3 would ideally be connected to all other faces, but since there is no real need for the connection in this work, face 3 is simply split in half and distributed to the top of faces 1 and 4 . The tops of faces 2 and 5 are filled as $\mathrm{NaN}$ (not a number) for computing purposes. One additional MATLAB specific issue results from the MATLAB matrix orientation relative to MITgcm orientation. In order to properly plot this data, it must be transposed first. This can additionally cause issues in calculations with 
derivatives. Care must be taken to ensure the correct direction is being used in MATLAB since $\mathrm{X}$ and $\mathrm{Y}$ directions are switched due to matrix orientation.

Depending on the resolution and variable being accessed, data files vary in both dimension and size. Table 1 shows some statistics for a typical data file. Taking the 1/48 degree data file for sea surface height as an example, there are about 260 million data points for each hourly output. Since the goal of this research is to work on time scales of months to years, efficient computation is essential.

The current high resolution model employs a Lat-Lon-Cap grid. This means that regions between $70 \mathrm{~S}$ and $57 \mathrm{~N}$ are simple latitude-longitude grids, but outside of those regions a "cap" is used. The cap is a spherical system used to help ensure a more constant grid spacing as longitude lines converge near the poles. This system ensures that all grid vertices occur over land and keeps cell sizes fairly constant (G. Forget, 2015).

Table 1: Variable statistics for Sea Surface Height.

\begin{tabular}{|l|l|}
\hline Common Name & Sea Surface Height \\
\hline Variable & Eta \\
\hline Size at $1 / 24^{\circ}$ & $8640 \times 7560$ \\
\hline Size at $1 / 48^{\circ}$ & $17280 \times 15120$ \\
\hline Nominal Resolution at $1 / 24^{\circ}$ & $\sim 4 \mathrm{~km}$ \\
\hline Nominal Resolution at $1 / 48^{\circ}$ & $\sim 2 \mathrm{~km}$ \\
\hline Time Resolution & $1 \mathrm{hour}$ \\
\hline Data File Size at $1 / 48^{\circ}$ & $1 \mathrm{~GB}$ \\
\hline Number of Files & $\sim 3000$ \\
\hline
\end{tabular}




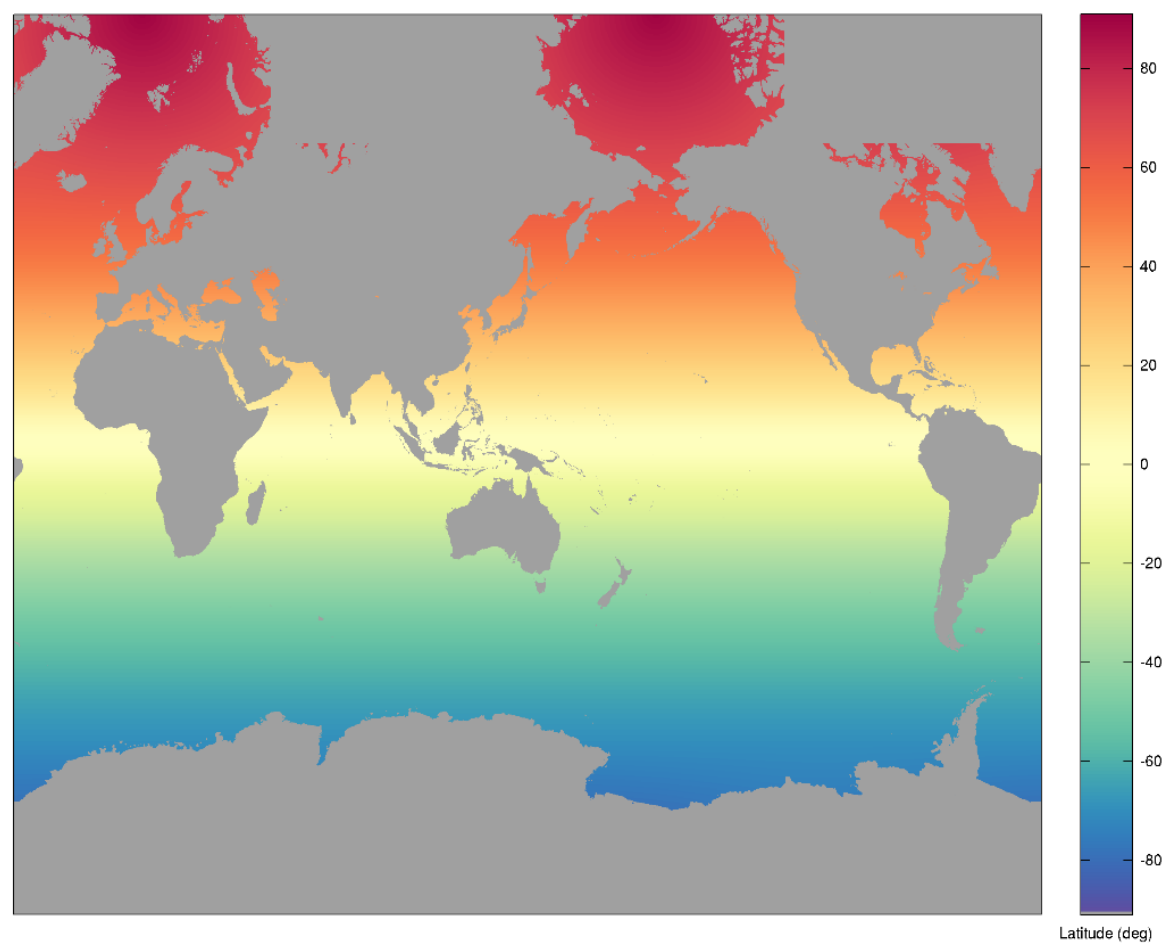

Figure 2: Latitude of grid cells in LLC-4320 1/48 degree model.

\subsection{Data}

All data for this work was taken from the $1 / 24^{\text {th }}$ and $1 / 48^{\text {th }}$ degree models. Table 2 shows the data sets used for each computation.

Table 2: Model variables used for computations in this work.

\begin{tabular}{|l|l|}
\hline Geostrophic & Eta, SIHeff, SNHeff, $x \mathrm{C}, \mathrm{yC}, \mathrm{dxC}, \mathrm{dyC}$ \\
\hline Ageostrophic & $\mathrm{U}, \mathrm{V}$, Geostrophic \\
\hline Ekman & oceTAUX, oceTAUY, yC \\
\hline Power & Geostrophic, Ageostrophic, Ekman, oceTAUX, oceTAUY, U, V \\
\hline General & landU, landV \\
\hline
\end{tabular}


Since this work is focused on surface currents and their respective wind work, the tides can provide false positive results. To filter out tides, a 30 hour low pass filter was used.

A digital windowing filter was designed for this purpose. Using a hamming filter of length 24 (in this case, length refers to the number of hours, since each data file corresponds to an hour time step), weights were chosen to result in a FIR (finite impulse response) low pass filter with a cutoff period of 30 hours and unity gain at DC. This filter allows for the filtering of tidal frequencies, which have peaks at 12 and 24 hours, with a bit of room to spare. Figures 3 and 4 show the phase and magnitude behavior of the chosen filter; note the linear phase and high attenuation in the stop band. Filter coefficients are shown in Table 3; here, note that the coefficients have been normalized to sum to one and that only the first twelve coefficients are shown due to symmetry.

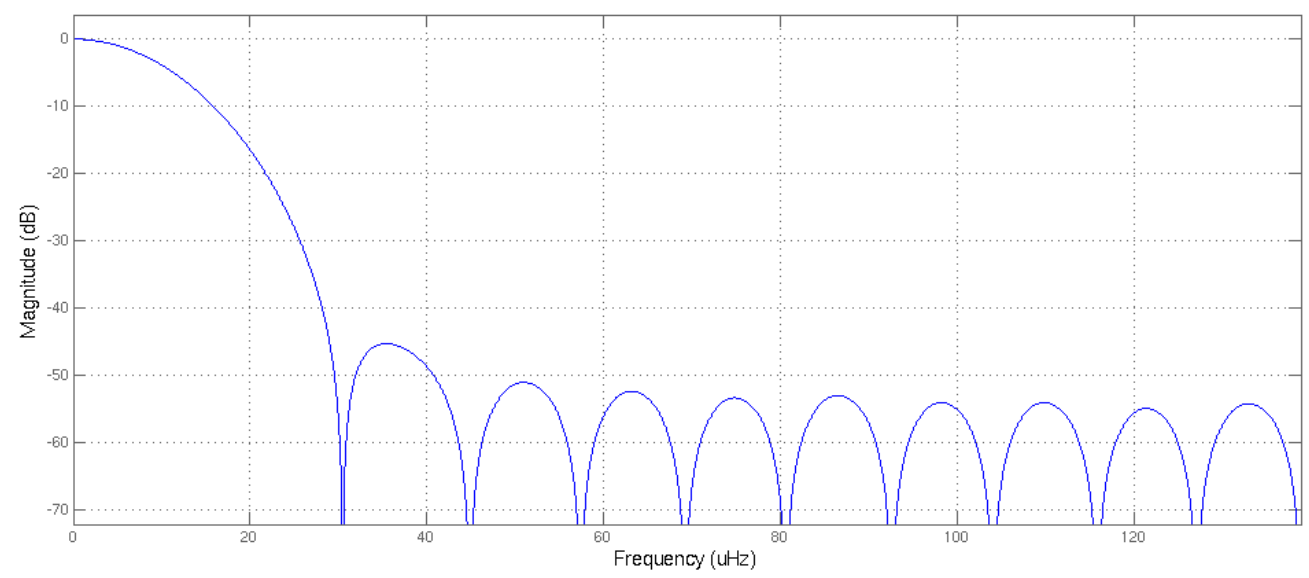

Figure 3: Magnitude response for 24 sample Hamming filter. 


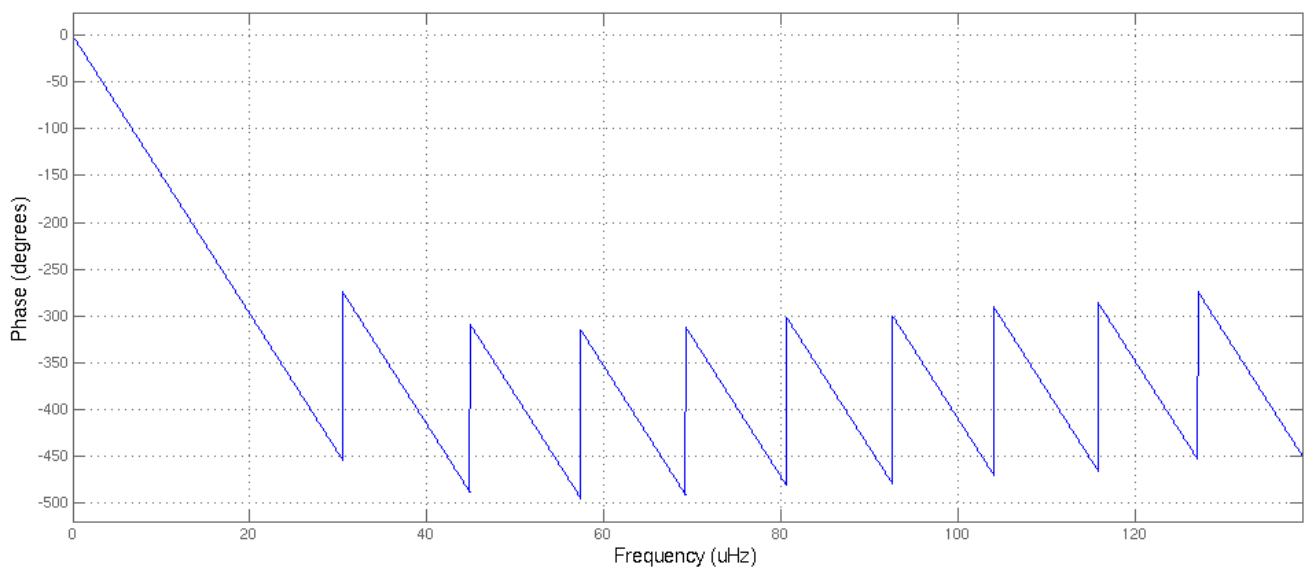

Figure 4: Phase response of 24 sample Hamming filter.

Table 3: Filter coefficients for 24 sample Hamming filter.

\begin{tabular}{|l|l|}
\hline Index & Coefficient \\
\hline 1 & .0021 \\
\hline 2 & .0033 \\
\hline 3 & .0063 \\
\hline 4 & .0116 \\
\hline 5 & .0195 \\
\hline 6 & .0299 \\
\hline 7 & .0423 \\
\hline 8 & .0556 \\
\hline 9 & .0686 \\
\hline 10 & .0799 \\
\hline 11 & .0883 \\
\hline 12 & .0927 \\
\hline
\end{tabular}


Low pass filtering was applied to raw model data of the following variables: sea surface height, ocean surface stress $\mathrm{U}\left(\tau_{\mathrm{x}}\right)$, ocean surface stress $\mathrm{V}\left(\tau_{\mathrm{y}}\right)$, total surface current $\mathrm{U}$, and total surface current $\mathrm{V}$. In reality, only the sea surface height requires low pass filtering, but filtering was applied to all data for consistency with previous studies.

In the Polar Regions, a floating layer of sea ice forms during the winter. This layer affects how sea surface height appears in the model, and therefore must be removed before computing currents based on sea surface height (geostrophic currents). This is done by equating ice height and density with a displacement due to ice. The same is done with snow, which accumulates on top of sea ice, all according to equations 2-3.

$$
\begin{gathered}
\text { Load }=H_{\text {ice }}^{\text {eff }} \times \rho_{\text {ice }}+H_{\text {snow }}^{\text {eff }} \times \rho_{\text {snow }} \\
S S H=\eta+\frac{\text { Load }}{\rho_{\text {ocean }}}
\end{gathered}
$$

Here, Load is the calculated ice load, $\mathrm{H}$ is the effective height of snow or ice (both given as variables in MITgcm), $\rho$ is the density of ice, snow, or ocean water, which are $910 \mathrm{~kg} / \mathrm{m}^{3}, 330 \mathrm{~kg} / \mathrm{m}^{3}$, and $1029 \mathrm{~kg} / \mathrm{m}^{3}$ respectively, $\eta$ is the given sea level, and $\mathrm{SSH}$ is the final calculated sea surface height.

This method works for most sea ice, but still more processing is necessary. To filter out the remainder of ice, a median filter is used on SSH. For regions below -65 degrees latitude and above 60 degrees latitude, a 25×25 cell standard deviation is taken at each point. Since ice corruption largely appears as areas of high standard deviation, points with large standard deviations have a 25x25 cell median filter applied to them. 
This, combined with the aforementioned ice+snow height method, effectively removes the majority of sea ice from the data.

Each step of ice removal is shown in Figures 5, 6, and 7. The original sea surface height data is shown first, in Figure 5. Sea ice appears in red, and is especially visible in the Northern Arctic regions, but also less so to the south. These areas of sea ice have currents flowing under the ice, albeit to a lesser extent than other regions of the world. Using the ice displacement calculation, outlined above, Figure 5 shows the first pass at ice removal. Regions that were significantly negative are now better behaved; some areas, however, display artifacts of ice removal. In the far Southern Ocean, in particular, areas where thin ice was removed appear pixelated. After using a standard deviation filter to detect these areas, a median filter is used to smooth out artifacts. These artifacts would cause issues down the road in calculating currents, since a derivative must be taken during calculations. 


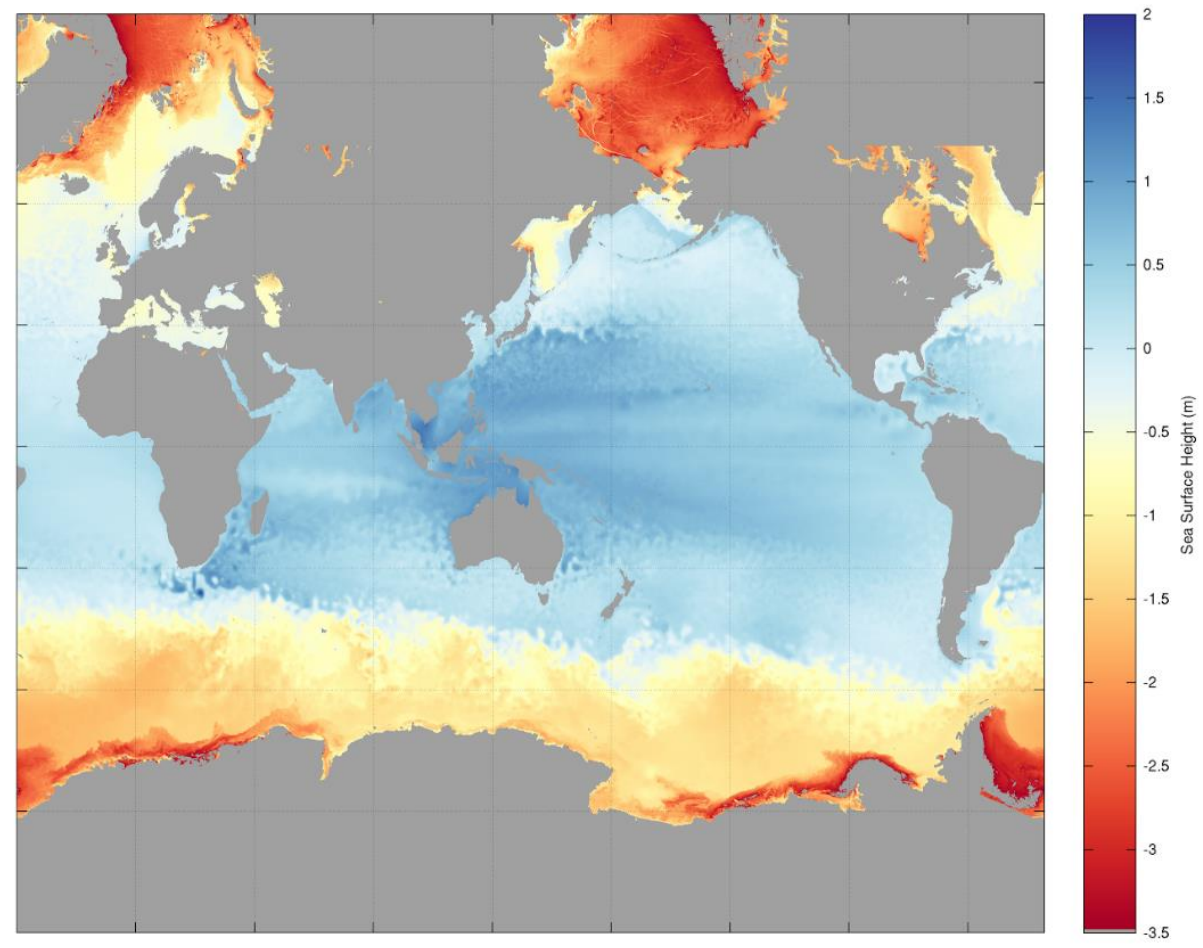

Figure 5: Original Sea Surface Height map with ice appearing in red.

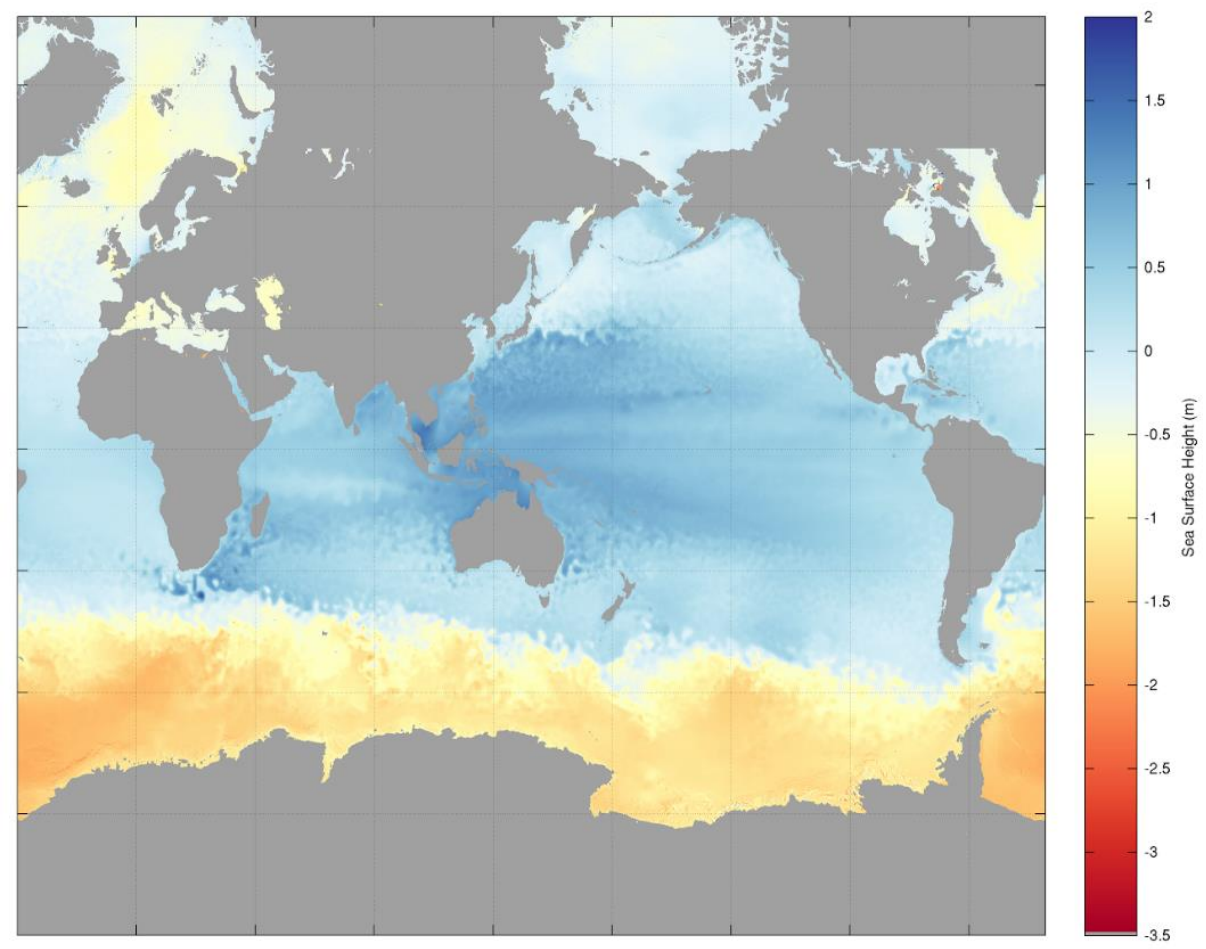

Figure 6: Sea Surface Height after first pass of ice removal. 


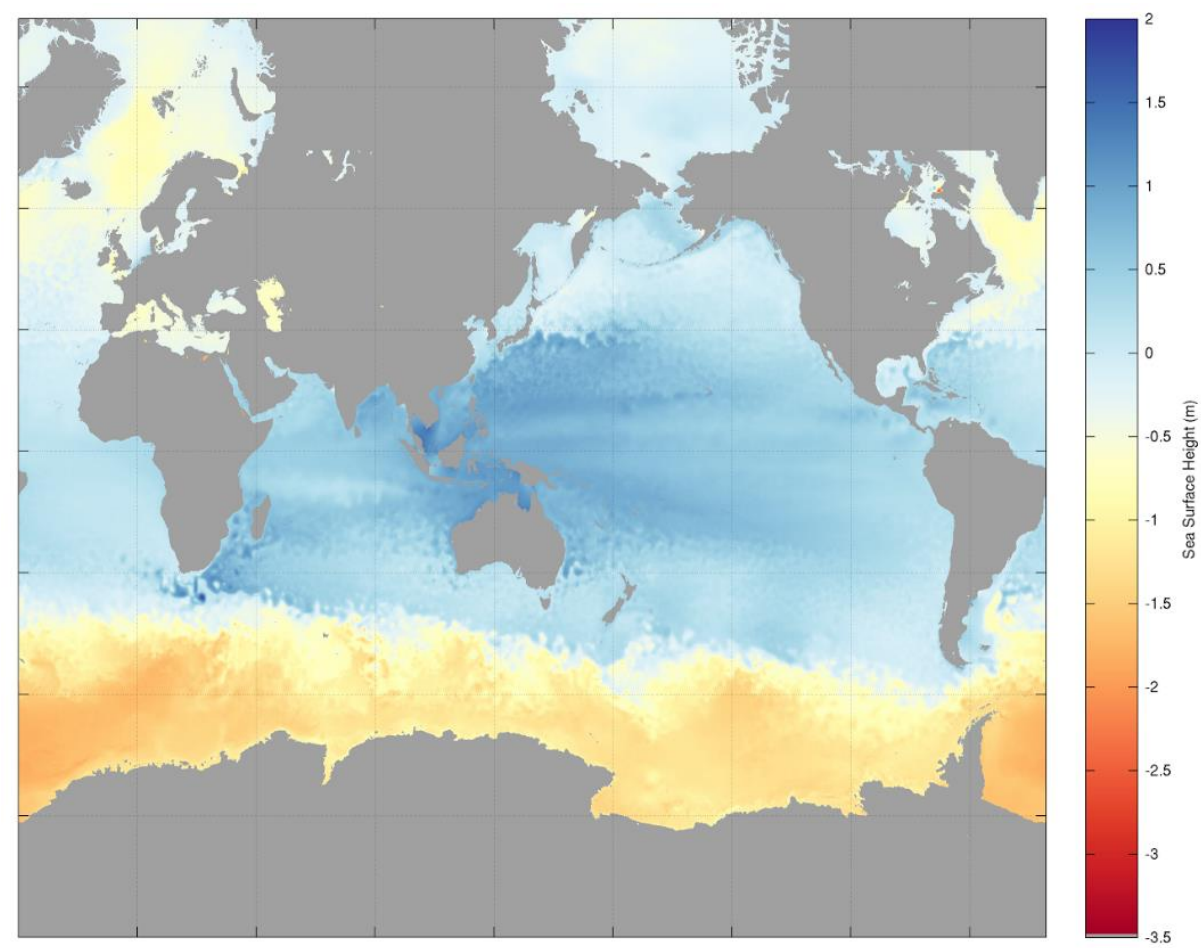

Figure 7: Final Sea Surface Height after filtering.

Finally, Figure 7 shows the end sea surface height product. This map shows an acceptable level of sea ice corruption.

\subsection{DopplerScat}

DopplerScat is a pre-proposal mission being investigated at the Jet Propulsion Laboratory/Caltech. The goal of the mission is to measure coincident total surface currents and surface winds. This will be accomplished via Doppler Scatterometry. With the measurement of both total surface currents and winds, the same calculations as are being done here with model data would be possible with real measured data. 
To help motivate the funding of DopplerScat, model data will be used to simulate DopplerScat observations. This will be done by adding predicted noise levels and filtering to achieve a similar resolution as DopplerScat. These simulated observations will be used to perform the same kinetic energy calculations as the original model data and comparisons will be made between the results.

\subsection{Time and Length Scales}

The model data set uniquely allows for very high resolution in both time and space. Two spatial resolutions will be considered: $1 / 48^{\text {th }}$ and $1 / 24^{\text {th }}$ degree models. These models are 2-5 times the resolution of the latest studies on wind work, which brings about unique issues both in terms of science and engineering. On the science side, spatial resolution could alter the results of this study compared to previous studies. At such high resolution, smaller surface current features that interact with the larger scale wind will be captured unlike previous calculations of wind work. In terms of engineering, such a high resolution presents an issue of processing. Optimized code in combination with NASA Advanced Supercomputing (NAS) made these computations possible. The same arguments can again be made for the time scales used. With hourly model output, much higher frequency features can be analyzed and must be filtered correctly. Most previous studies have used satellite data that takes about a week to fully scan the Earth, which means only the lower frequency features can be captured.

Since model data can be used at nearly any time scale (with appropriate averaging), multiple averaging periods will also be considered. This will help validate calculations against previous studies and also help to better explain interactions between 
surface wind and currents. The one day hamming filtered data will be averaged for 3, 7 , 11 , and 15 day averages to achieve a wide range of time scales. 


\section{CHAPTER 2 ANALYSIS}

\subsection{Currents}

The goal of this work is to investigate the energy exchange between the wind and the upper ocean. Towards that end, a number of beginning and intermediate steps must be taken to prepare for energy calculations. First, data must be compiled, filtered, and saved in a useable format. Then, different types of surface currents must be calculated. Finally,

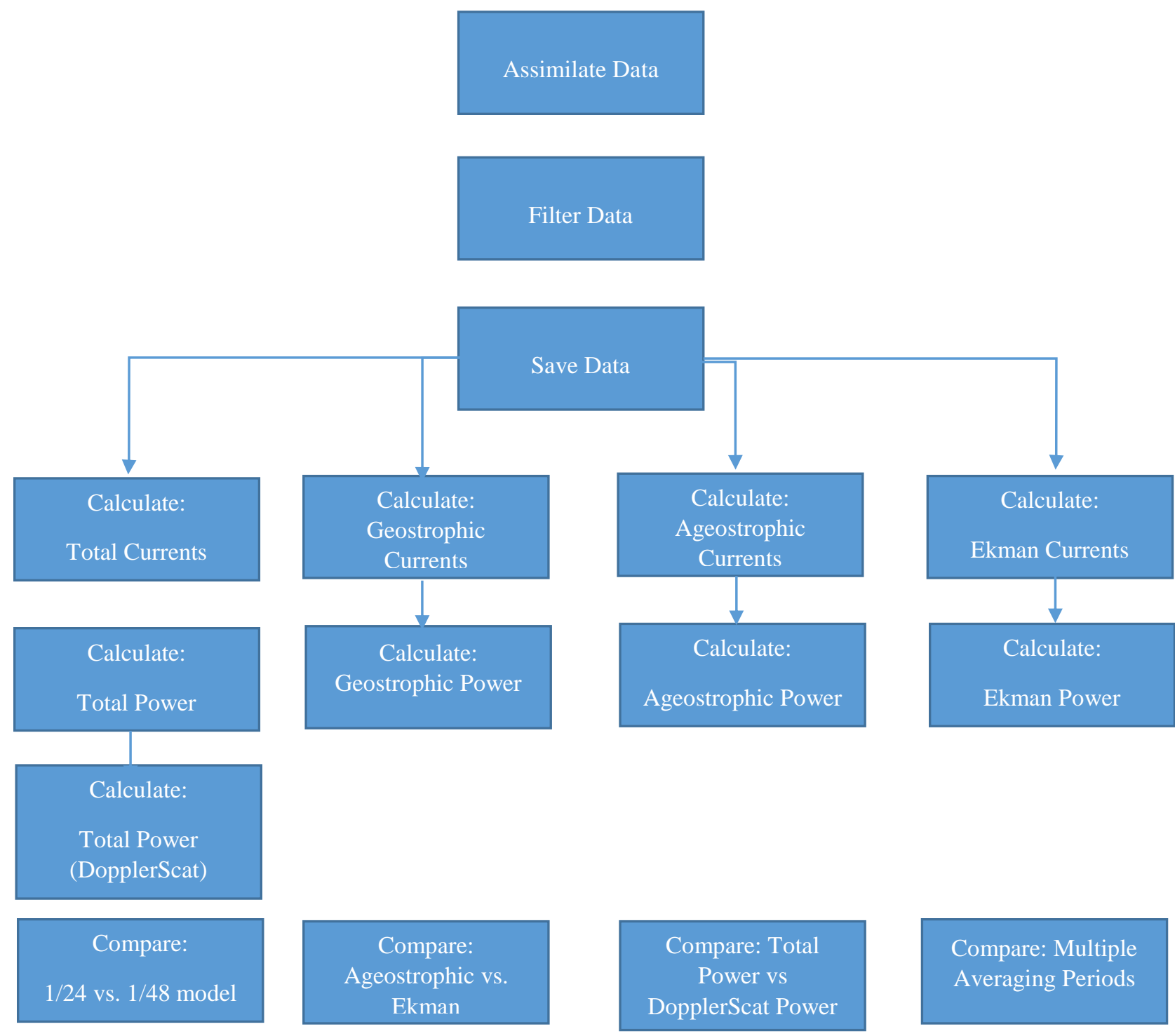

Figure 8: Process diagram of work done in this research. 
the wind power exchange for each type of surface current is calculated. A flow chart of this sequence is shown in Figure 8.

In general, the kinetic energy flux at the ocean surface may be calculated according to the equation,

$$
\dot{K E}=\vec{U} \cdot \vec{\tau},
$$

where $\vec{U}=(\mathrm{u}, \mathrm{v}, \mathrm{w})$ is the current vector at the ocean surface, and $\vec{\tau}=\left(\tau_{\mathrm{x}}, \tau_{\mathrm{y}}\right)$ is the ocean surface wind stress (Wunch, 1998). In the MITgcm model, wind stress is calculated using the bulk formula,

$$
\vec{\tau}=\rho_{a i r} C_{d}\left|\vec{U}_{10}-\vec{U}_{s}\right|\left(\vec{U}_{10}-\overrightarrow{U_{s}}\right)
$$

where $\rho_{\text {air }}$ is the density of air, $C_{d}$ is the drag coefficient, and $U_{10}$ is the 10 meter wind speed, an approximation for the surface wind speed. In general, $C_{d}$ is on the order of $10^{-3}$, but this implementation of MITgem uses a varied $C_{d}$ based on model stability and surface wind speed (Menemenlis, personal communication). Wind speeds from the European Center for Medium Range Weather Forecasting are used in this MITgcm model, interpolated to a $1 / 48^{\text {th }}$ degree grid.

An important distinction should be made here between this work and many previous studies. The majority of studies have used a definition of surface wind stress that neglects the surface current contribution $\left(\mathrm{U}_{\mathrm{s}}=0\right)$. Using the above definition is more accurate and results in a significantly lower computed power (Zhai, 2012). Setting $U_{\mathrm{s}}$ to zero is sometimes called the "resting ocean approximation." 
Depending on the type of currents being used, the calculations for $U_{s}$ vary, as defined below.

\subsubsection{Geostrophic Currents}

Geostrophic currents are formed by the balance of surface pressure gradients and the Earth's rotation. This type of current flows along isobars due to the aforementioned balanced forces. The equations that describe these currents may be derived from a momentum balance and are defined in equations 6 and 7. These equations make the assumptions that the friction term in the Navier-Stokes equations can be neglected and that the Coriolis effect is non-negligible compared to the acceleration term.

$$
\begin{gathered}
U_{s}=-\frac{g}{f} \frac{\partial \eta}{\partial y} \\
V_{s}=\frac{g}{f} \frac{\partial \eta}{\partial x}
\end{gathered}
$$

Here, $U_{s}$ and $V_{s}$ are the zonal and meridional components of surface currents (along the $\mathrm{X}$ and $\mathrm{Y}$ directions respectively). The $g$ term is the acceleration due to gravity $\left(9.81 \mathrm{~m} / \mathrm{s}^{2}\right), \frac{\partial \eta}{\partial y}$ and $\frac{\partial \eta}{\partial x}$ are the partial derivatives of sea surface height in the meridional and zonal directions respectively - the sea surface slope. The Coriolis parameter, $f$, is calculated based on the local latitude,

$$
f=2 \Omega \sin (\lambda)
$$


where $\lambda$ is the latitude and $\Omega$ is the rotation rate of Earth. In performing this geostrophic calculation, globally gridded $\left(1 / 48^{\text {th }}\right.$ and $1 / 24^{\text {th }}$ degree $)$ daily averages of sea surface height were initially used, followed by $3,7,11$, and 15 day averages. Since $f$ goes to zero at the equator, the geostrophic equations break down at zero latitude. For this work, the center three degrees latitude were omitted (but averaged over) in power summations.

Geostrophy makes the assumption that currents are actually in geostrophic balance - which is not necessarily the case. Some regions of the ocean are more prone to geostrophic balance than others due to frictional effects, for example. Geostrophy also requires certain length and time scales to be valid. The Rossby number is sometimes used as a guideline for whether geostrophy is applicable on the scales observed.

$$
R=\frac{U}{L f}
$$

In this case, the Rossby number is calculated using the resolution cell size (L), order of magnitude surface currents $(\mathrm{U})$, and the range of Coriolis parameters $(f)$. This yields Figure 9. Geostrophic balance is considered a reasonable assumption for Rossby numbers much less than 1. The majority of the ocean in Figure 9 is just barely in the realm of geostrophy. Ideally, the Rossby number would be closer to .1, but higher resolution and geostrophic balance are inversely related. Achieving the high resolution desired in this study means standing on the edge of geostrophy. A similar argument for time scales can be made with geostrophic currents. On too short a time scale, geostrophic currents may not be visible (a few days may be required). This will become apparent in the different averaging schemes used. 


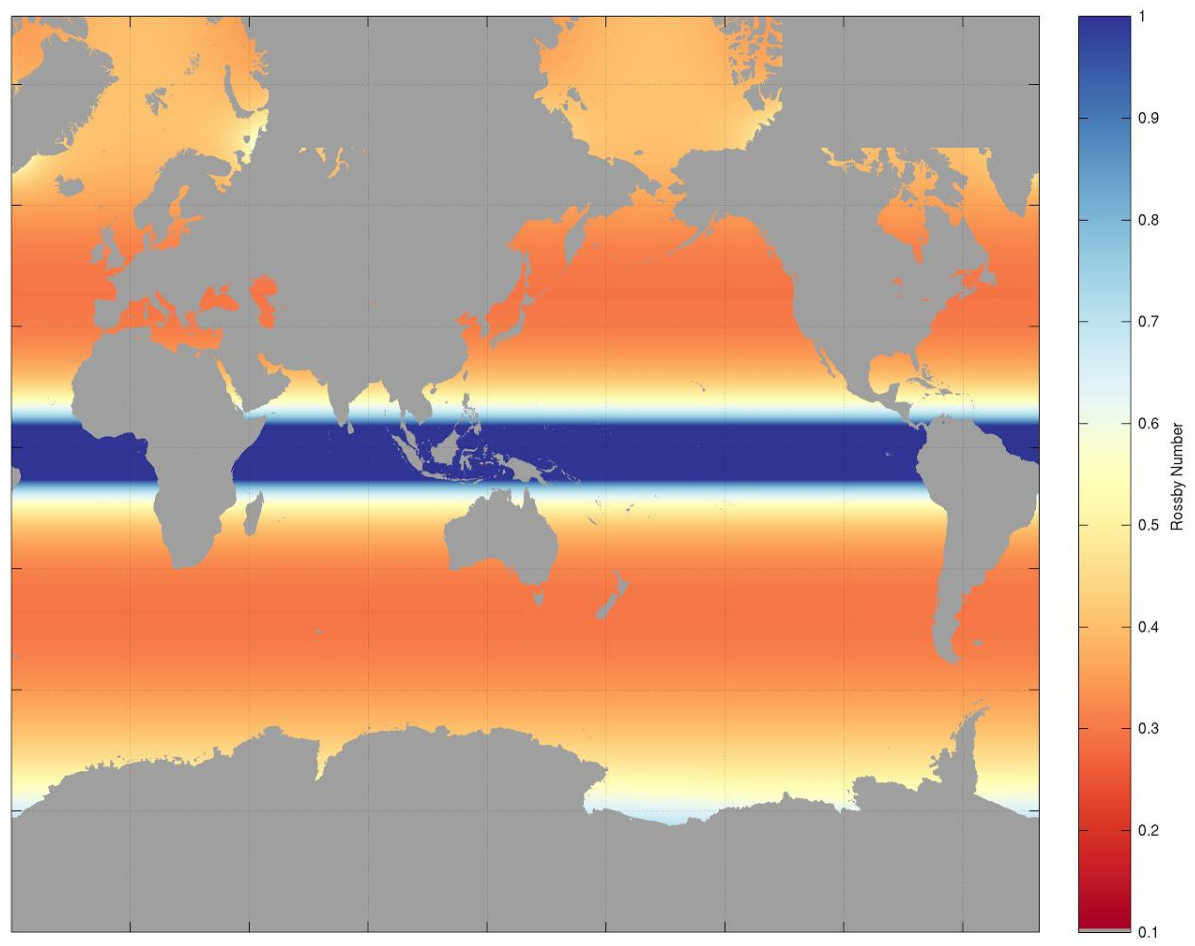

Figure 9: Rossby number for $1 / 48$ degree model.

\subsubsection{Ageostrophic Currents}

Ageostrophic currents are the catch all for any type of current that is not in geostrophic balance. These types of currents are largely wind-driven and can account for the majority of currents in the upper 100 meters of the ocean. By summing the ageostrophic and geostrophic components of surface currents, the total ocean currents may be calculated. Conversely, as will be done in this work, ageostrophic currents may be calculated based on the difference between total (as output by MITgcm) and geostrophic currents.

$$
\mathrm{U}_{\mathrm{T}}=\mathrm{Ug}+\mathrm{Uag}
$$


Problematically, geostrophic currents are not as explicitly defined as their geostrophic counterparts. There is not a direct way of calculating them, short of taking into account every possible input into the upper ocean. For this reason, geostrophic currents are sometimes approximated as their primary component: Ekman currents.

\subsubsection{Ekman Currents}

Ekman currents are the result of the balance between surface wind stress (the friction due to wind on the ocean surface) and the Coriolis force. This force balance creates surface currents that theoretically travel 45 degrees to the right of the wind direction (to the left in the Southern Hemisphere). This work relies on the assumption that resulting Ekman currents will be at a 45 degree angle to the wind, an assumption that is known to not be entirely accurate in reality (Wang, 2003). Surface Ekman currents may be calculated according to Equation 11,

$$
\left|U_{e k}\right|=\frac{|\tau|}{\sqrt{\rho^{2} A_{z} f}}
$$

where $|\tau|$ is the surface stress magnitude, $\rho$ is the density of sea water, $A_{z}$ is the vertical viscosity, and $f$ is the Coriolis parameter. With the magnitude of $\mathrm{U}_{\mathrm{ek}}$ calculated, $\mathrm{U}_{\mathrm{ek}}$ is then rotated 45 degrees to the right of the wind vector in the Northern Hemisphere (to the left in the Southern Hemisphere). Most of the parameters in Equation 11 can be calculated from MITgcm output, except for Az. Vertical viscosity $\left(\mathrm{A}_{\mathrm{z}}\right)$ is a mixing parameter that varies throughout the ocean and is not well parameterized. 
In order to find a suitable value for $\mathrm{A}_{\mathrm{z}}$, optimization was used. For a 10 degree patch in the mid-North-Atlantic Ocean, both geostrophic and Ekman currents were calculated. By subtracting these geostrophic currents from the total MITgcm currents, ageostrophic currents are left over. $\mathrm{A}_{\mathrm{z}}$ was then optimized to result in the minimum difference between ageostrophic currents and calculated Ekman currents. Constrained optimization was used with Sequential Quadratic Programming. This resulted in an optimal value of .0104 for $A_{z}$, as shown in Figure10. $A_{z}$ does vary throughout the ocean, so this single optimum does not truly apply globally. A single value is still used, though, because extending the optimization routine to the entire ocean would be too costly for this study.

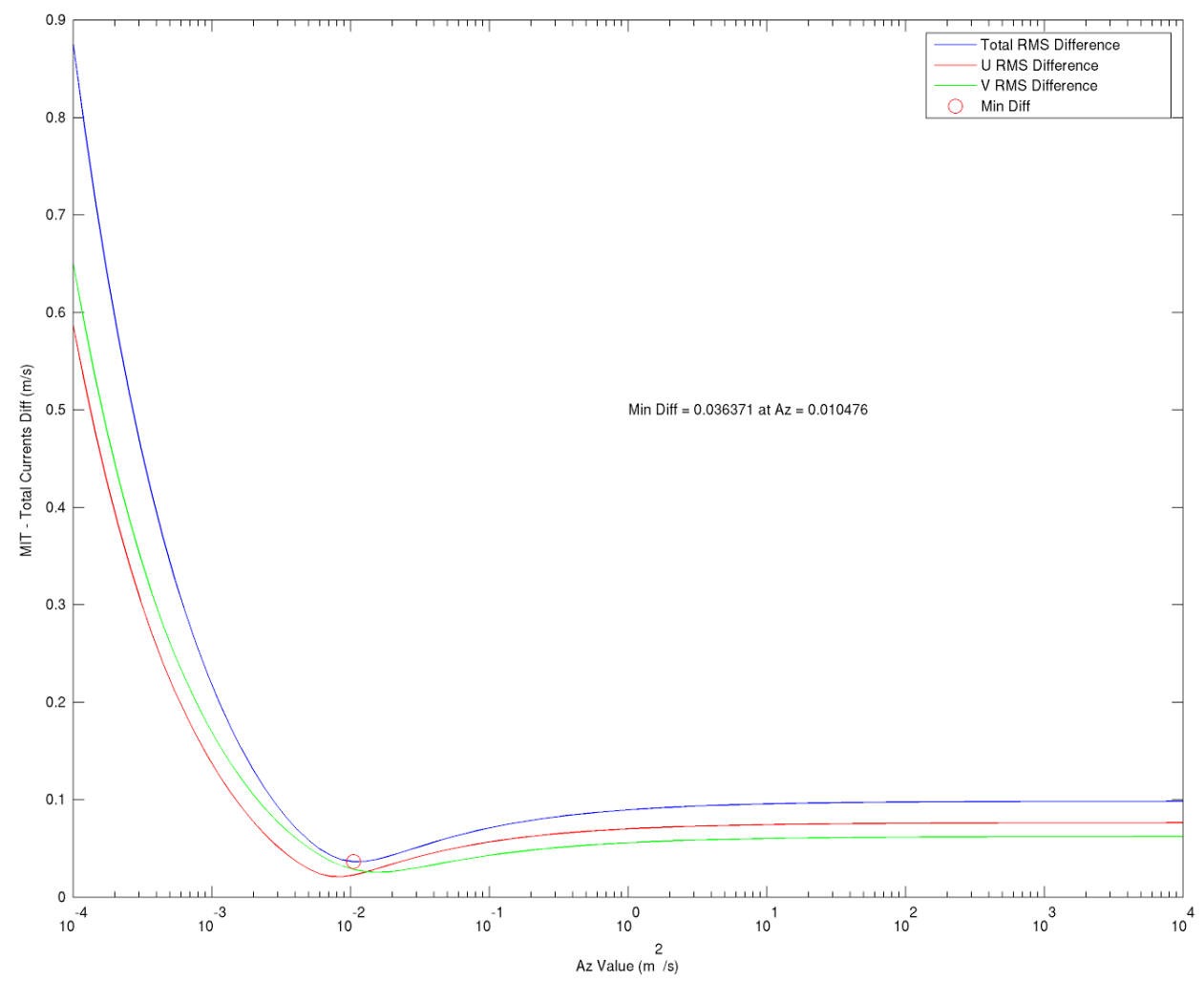

Figure 10: Optimization for vertical viscosity results in Az of .014. 


\subsection{Kinetic Energy Flux and Power}

With the right data, wind driven kinetic energy flux is not a difficult calculation. In fact, it requires only the dot product between surface wind stress and surface currents.

$$
F_{T}=\vec{U} \cdot \vec{\tau}
$$

Where $\mathrm{U}$ and $\tau$ are the surface current and wind stress vectors. The surface current vector could be any of the previously mentioned surface currents: geostrophic, ageostrophic, or Ekman. By splitting up each of these current types, the amount of kinetic energy flux due to each may be found.

In order to determine the total power, each cell's flux would ideally be multiplied by its area and then summed.

$$
P=\sum_{i=1}^{M} \sum_{j=1}^{N} A_{i j} F_{T i j}
$$

Here, $\mathrm{P}$ is the total instantaneous kinetic energy transferred, or power at the current time step, A is the area of each cell, and F is the kinetic energy flux through each cell. $\mathrm{M}$ and $\mathrm{N}$ are the matrix dimensions for the map being used.

Since areas of the ocean are corrupted occasionally and the equatorial region is omitted in all geostrophic calculations, kinetic energy flux is averaged and then multiplied by the total relevant ocean area. This achieves essentially the same end result as the summation method, but allows for areas with little or no data to still be included in the result. 


\subsection{DopplerScat}

Two main features separate MITgcm output and nominal DopplerScat observations: noise and resolution. DopplerScat is set to achieve approximately $10 \mathrm{~km}$ resolution, with $1.1 \mathrm{~m} / \mathrm{s}$ wind speed noise and $.5 \mathrm{~m} / \mathrm{s}$ current noise. This means that zero mean Gaussian noise must be added to model data and a filter must be applied to reduce the $\sim 2 \mathrm{~km}$ resolution. Once these two changes are made, power computations can be made on the transformed data to see how DopplerScat would see wind power input.

First, a blurring filter is applied to MITgcm output of wind stress and total surface currents. This blurring filter consists of a 2-D variable length Gaussian filter, convolved with the desired MITgcm map. A varied length is used depending on the resolution of the cell being converted, and is required because MITgcm LLC gridding is not constant in size. By varying the filter length, the correct number of pixels are averaged together each time. To implement this varied filter length, zonal (latitudinal) strips were filtered sequentially with a 2-D Gaussian filter of length determined by the resolution in the relevant strip. Filter length was chosen based simply on the desired resolution of $10 \mathrm{~km}$ and the current resolution. For example, if the strip in question has an average resolution of $2 \mathrm{~km}$, then the filter length would be chosen as $10 / 2=5$ grid cells. While not optimal, this rough filter length estimate is better than a blanket filter length.

The original proposal for this work involved using a constant length 1D Parzen filter, applied to both rows and columns, for filtering. In theory, this would work, but with tens of thousands of columns and rows, such a computation would take a very long time. Since Parzen filters are essentially Gaussian approximations, a 2D Gaussian filter was used instead and resulted in a significant reduction in computation time. A value of 
2.5 was chosen for sigma in the Gaussian filter since it closely matched a Parzen filter of the same length.
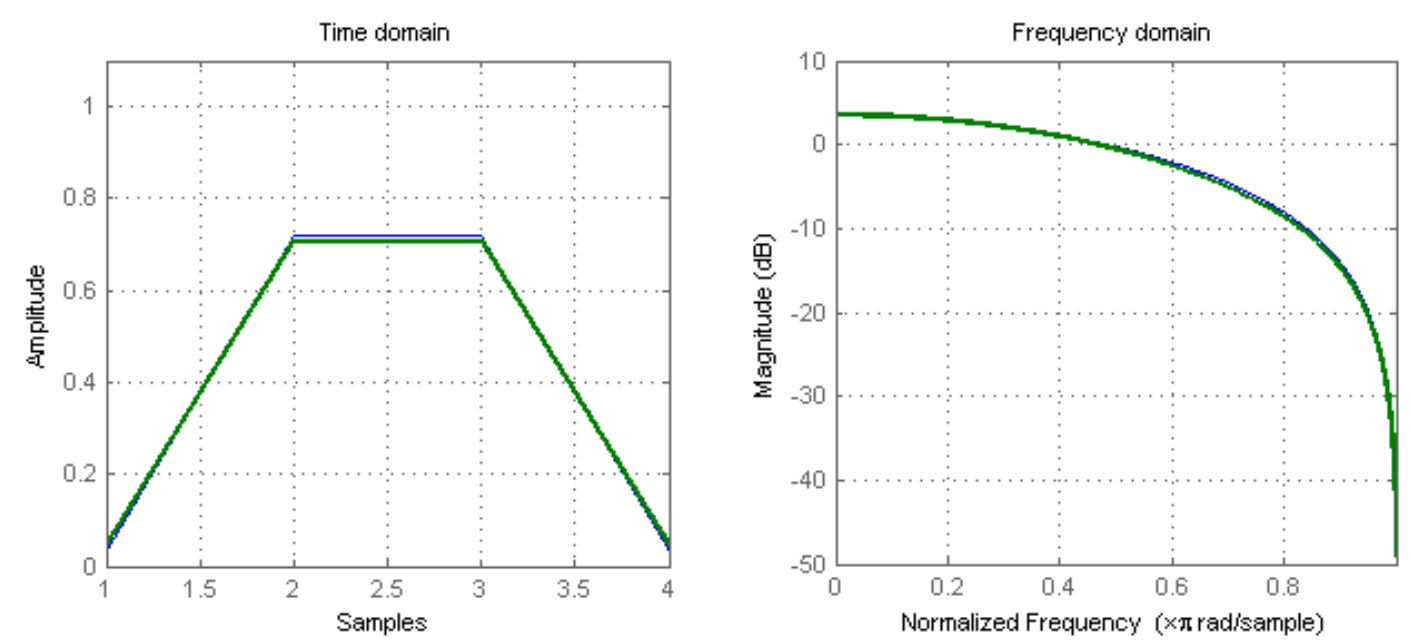

Figure 11: Comparison of Parzen and Gaussian filters of length 4.

This method, of course, does not result in a true reduction in resolution. There will still be the same number of pixels in the end product, but the effective resolution is decreased. To truly reduce the resolution, down sampling is used after filtering.

Adding noise is relatively simple compared to reducing the varied cell size. Once cell sizes have been blurred, they are down sampled to the correct resolution and zero mean white Gaussian noise is added with standard deviation of $1.1 \mathrm{~m} / \mathrm{s}$ in wind and $.5 \mathrm{~m} / \mathrm{s}$ in surface current. These standard deviations were taken from DopplerScat system requirements. Since DopplerScat system requirements specified noise in terms of wind speed and MITgcm only provides wind stress, the surface stress was converted to wind speed before adding wind-noise, and then back again to wind stress for power 
computations. This conversion was done with the bulk formula given in equation 5 , assuming constant drag coefficient and $\mathrm{U}_{\mathrm{s}}$ of zero.

With filters and noise set up, the actual data used for these DopplerScat computations is important. Unlike previous data sets, this data will not be averaged in an attempt to better match DopplerScat observations. Since DopplerScat will scan the entire earth almost daily, single hourly snapshot data will be used on a daily basis. This means that only one snapshot of the ocean surface will be taken daily to compute wind work. From these computations, a seven day average is taken to see how well DopplerScat can resolve wind work with seven days of surface stress and current averaging, calculated from daily snapshots. 


\section{CHAPTER 3 LITERATURE REVIEW}

As far back as the 1960s (and probably earlier), oceanographers have asked the question: how much energy is exchanged between the wind and ocean surface currents? A number of findings suggest a geostrophic kinetic energy flux of about 1 TW (Faller, 1968; Fofonoff, 1981; Oort, 1994; Wunch, 1998). More recently, Scott and Xu estimate the geostrophic kinetic energy flux at between .85 and 1 TW (Scott, Xu, 2008). The above calculations all match fairly well, largely because they are all essentially the same calculation done with different data sets. In the last few years, new estimates of the geostrophic kinetic energy flux have cut the energy input significantly. Zhai et al. (2012), using a $1 / 3^{\text {rd }}$ degree TOPEX reanalysis, estimated the geostrophic wind work to be closer to $.43 \mathrm{TW}$, a difference primarily attributed to wind stress averaging period and taking into account what Zhai calls the "resting ocean approximation," used in the previous studies. Zhai found that using daily wind stress means, rather than the weekly or monthly wind stress means used in previous studies, the resulting power input was significantly larger. These geostrophic calculations, while all correct in their own sense, leave out a significant part of the story. Ageostrophic currents account for a large portion of surface currents, and, correspondingly, a large portion of kinetic energy flux.

Ageostrophic currents are far less studied than their geostrophic counterparts particularly from an energetics standpoint. Wang and Huang estimate the ageostrophic kinetic energy flux to be about 2.4 TW. This was done with a 1.875 degree wind stress model and by approximating the ageostrophic current component using simple Ekman dynamics (Wang and Haung, 2003). While this approximation is likely a good one, there 
are other contributors to ageostrophic currents. Since a full ocean model will be used in this work, this Ekman approximation can be tested.

By employing a $1 / 10^{\text {th }}$ degree $(\sim 10 \mathrm{~km})$ general circulation model, Von Storch et al. (2006) was able to globally compute the total surface kinetic energy flux. Von Storch estimated the total wind generated power at the sea surface to be about $3.8 \mathrm{TW}$ (Von Storch et al., 2006). That is, theoretically, the combination of both geostrophic and ageostrophic currents, or,

$$
F_{T}=F_{g}+F_{a g}
$$

where F represents surface kinetic energy flux, and T, G, and AG represent total, geostrophic, and ageostrophic components respectively. Summing the aforementioned geostrophic and ageostrophic fluxes of $1 \mathrm{TW}$ and 2.4 TW yields $3.4 \mathrm{TW}$ - quite close to the Von Storch results.

This work will aim to similarly calculate the total kinetic energy flux, but instead will use a higher resolution (in both time and space) general circulation model, and will additionally separate the geostrophic and ageostrophic components. These results will be applied to simulating satellite observations, used to help explain differences between previous studies, and used to motivate the funding of a Doppler Scatterometer instrument, DopplerScat. 


\section{CHAPTER 4 DISCUSSION}

\subsection{Currents}

\subsubsection{Total Currents}

Total currents are one of the variables output by the MITgcm model. Seven days of currents during November 2012 were averaged to produce the map shown in Figure 12 , where magnitude is represented by the red color scale and vectors show direction and magnitude. Many of the prototypical currents are visible. The western boundary currents flow up and along the eastern coasts of the United States and Asia, while the highly active Southern Ocean is filled with eddies. The Antarctic Circumpolar Current (ACC) flows eastward with strong jets that are clearly visible as dark red on the color scale. Near the equator, the Intertropical Convergence Zone (ITCZ) generates currents that

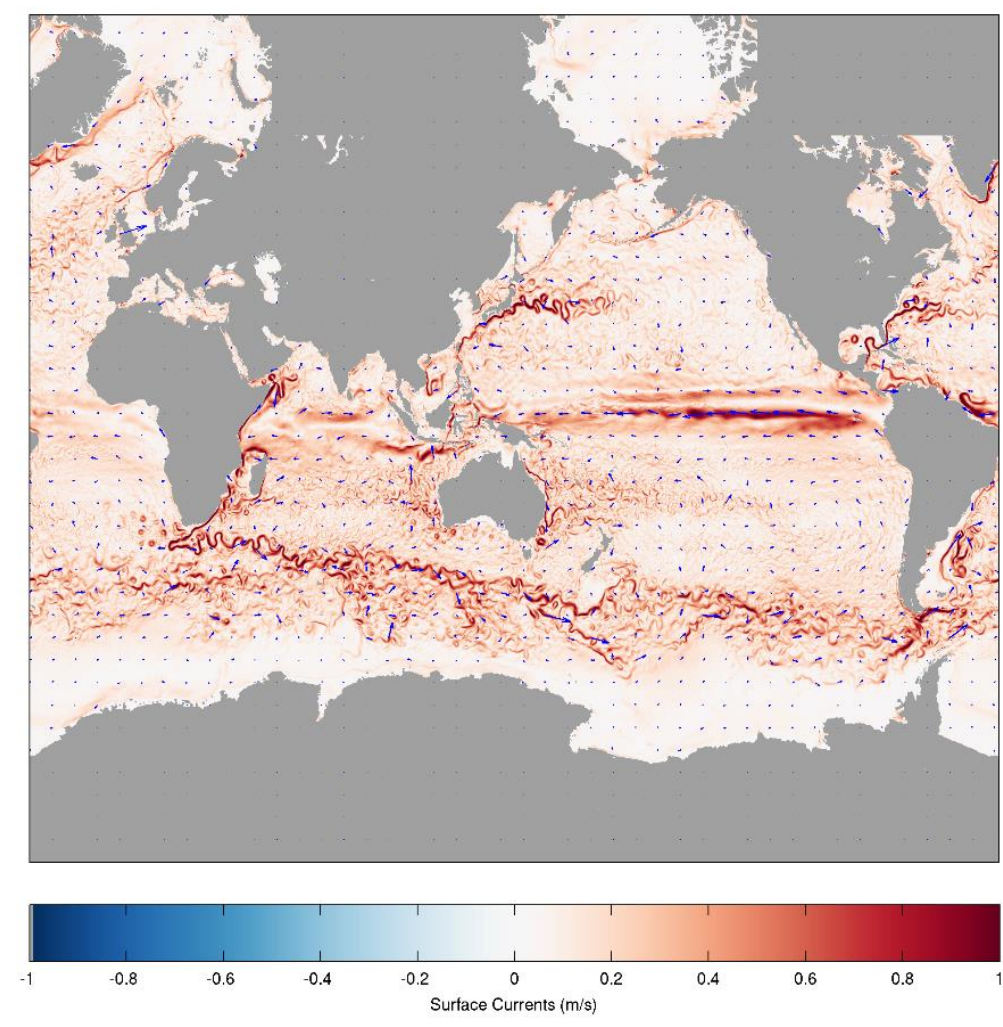

Figure 12: Total currents as output by the MITgcm model. Seven day average during Nov 2012. 
consistently flow towards the west. These total model currents match up well with the available observations and theory.

\subsubsection{Geostrophic Currents}

Geostrophic currents were calculated based on Sea Surface Height (SSH). Shown in Figure 13, geostrophic currents match up well with many of the strong, mesoscale currents in Figure 12. The western boundary currents along the costs of the United States and Asia and the jets in the ACC show particularly strong signals. As expected, the geostrophic computations break down along the equator causing large, positive currents to be calculated - these are not correct, and thus the center 6 degrees latitude are omitted. Geostrophic currents flow along isobars, which are approximated here using sea surface

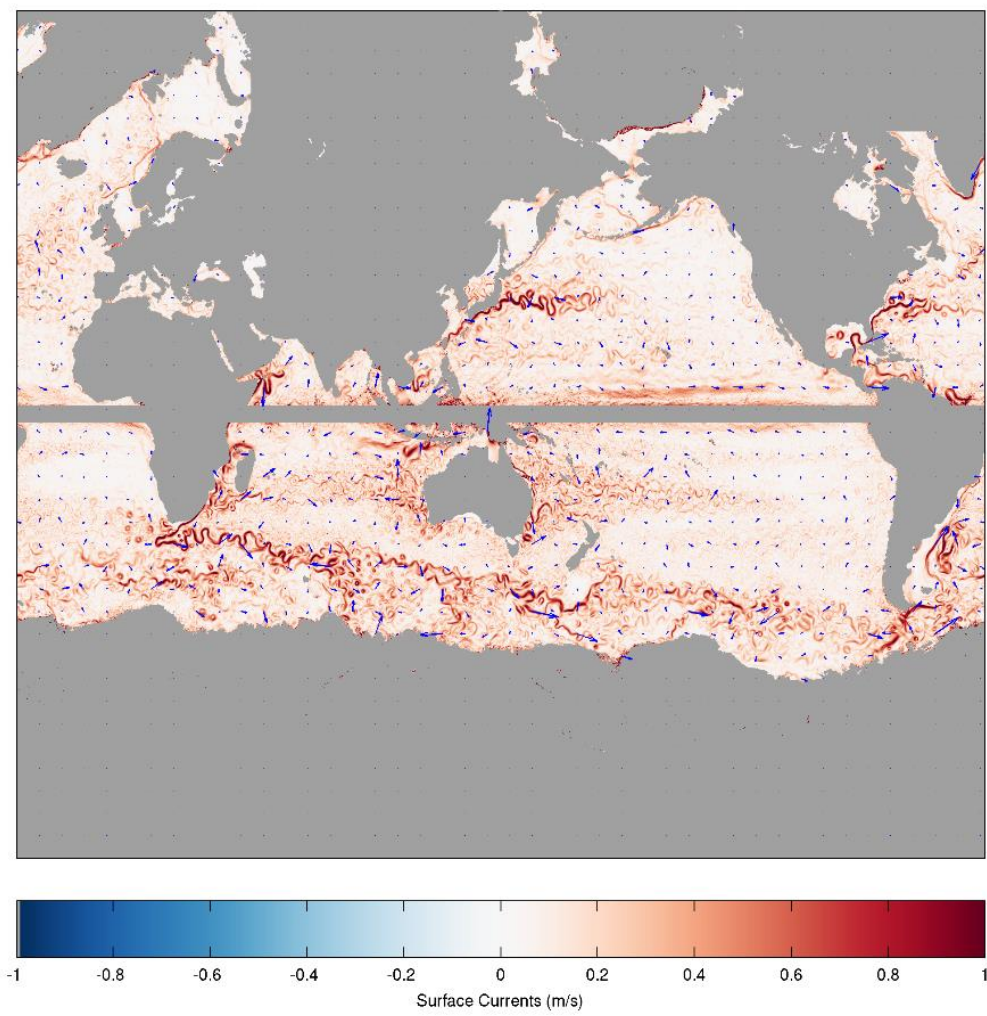

Figure 13: Geostrophic currents as calculated based on a seven day average of Sea Surface Height during Nov 2012. 
heights. Figure 14 shows the geostrophic flow, clearly moving around peaks and troughs in the sea surface height.

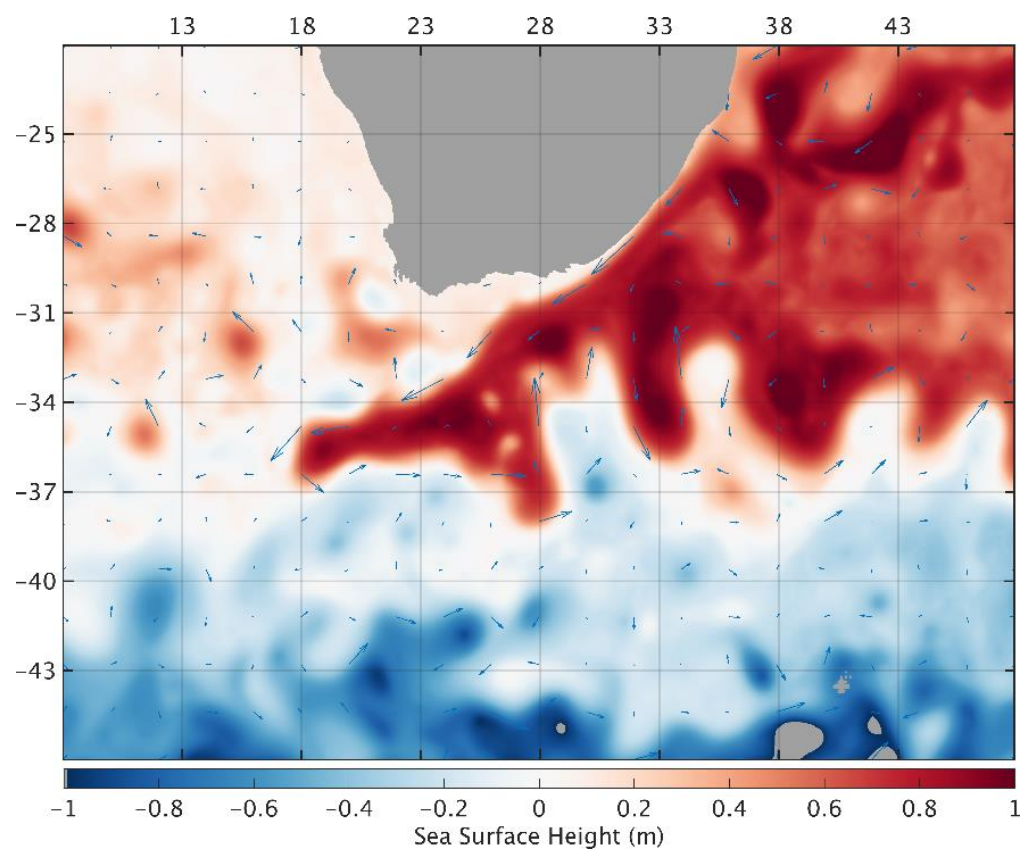

Figure 14: Geostrophic currents plotted over sea surface height in the Southern Ocean off the tip of Southern Africa. Seven day averages shown.

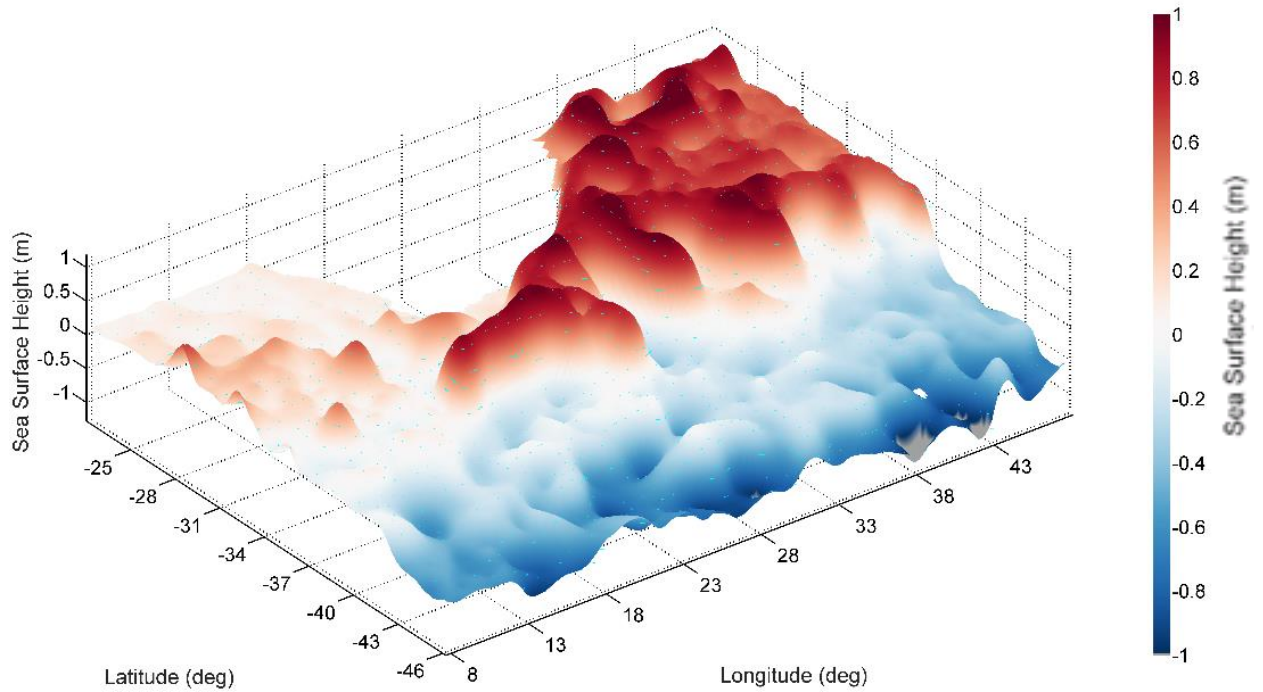

Figure 15: Geostrophic vectors plotted over 3D sea surface height data. Again, off the tip of Southern Africa with 7 day averages. 


\subsubsection{Ekman Currents}

Ekman currents were calculated based on surface wind stress and simple Ekman dynamics (45-degree angle). Plotted in Figure 16 are Ekman currents during November 2012, calculated from a seven-day average of surface wind stress. Since winds act on a much larger scale than do geostrophic currents, so do Ekman currents act on a larger scale. Notice the large-scale anti-cyclone off the western coast of South America and the general eastern directed flow in the ACC. These currents appear much smoother than the total or geostrophic currents primarily due to the large-scale wind, but also partially because of the smooth wind vectors output by ECMWF.

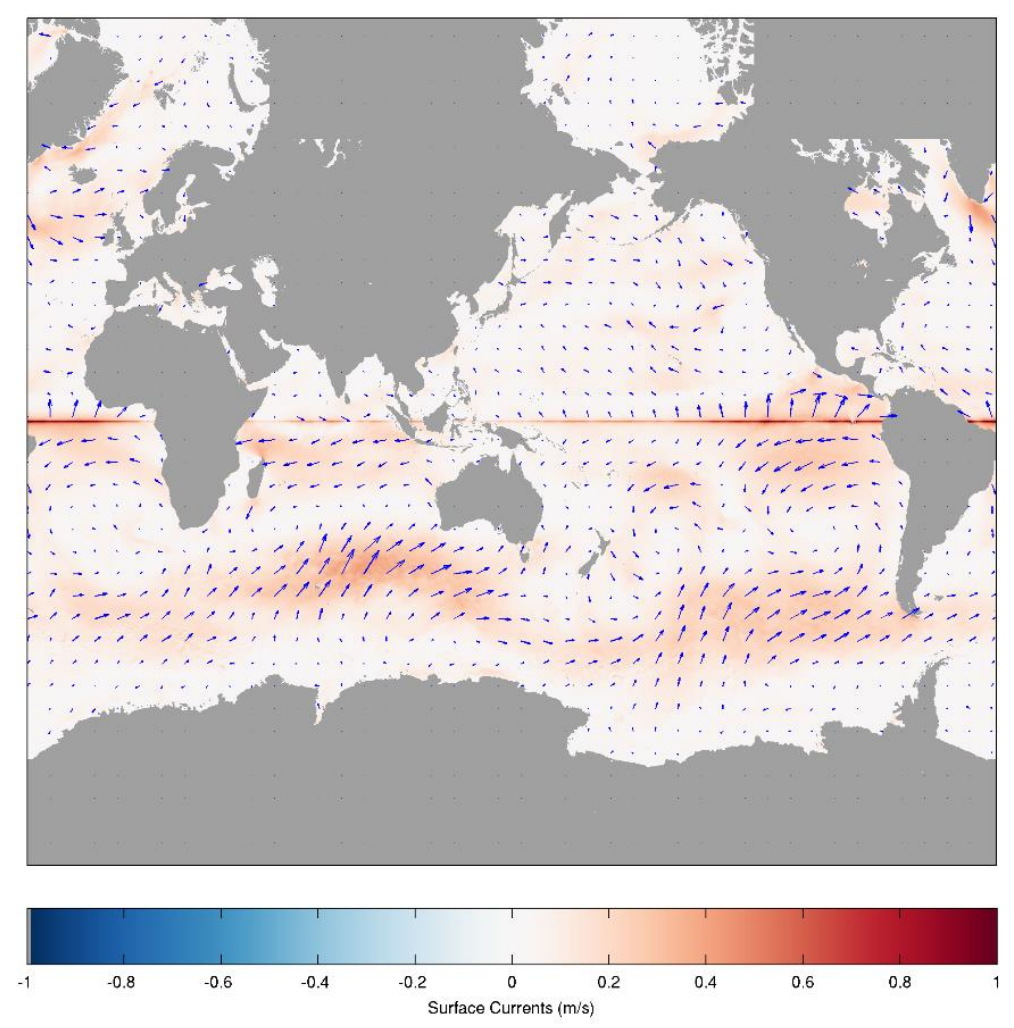

Figure 16: Ekman currents as calculated based on a seven day average of surface stress during Nov 2012. 


\subsection{Power}

All power and flux calculation results shown in this section are were made according to the procedures shown in the analysis section. Averaging was set at seven days for surface current calculation and one day for wind stress to better match up with previous studies.

\subsubsection{Total (MITgcm) Power}

Figures 15 and 16 show a seven day average of total surface currents, a one day average of surface stress, and total kinetic energy flux, calculated from the previous two. Since KE flux is calculated as the dot product between surface stress and surface currents, areas with wind stress and surface currents oriented in the same direction will produce positive KE flux. Opposing currents and stress will generate negative KE flux (ocean does work on the atmosphere). With this in mind, consider Figure 17. Global surface currents and surface wind stress generate significant, positive work globally about 3.2 TW on average from September 2011 through February 2012 in the $1 / 48^{\text {th }}$ degree model.
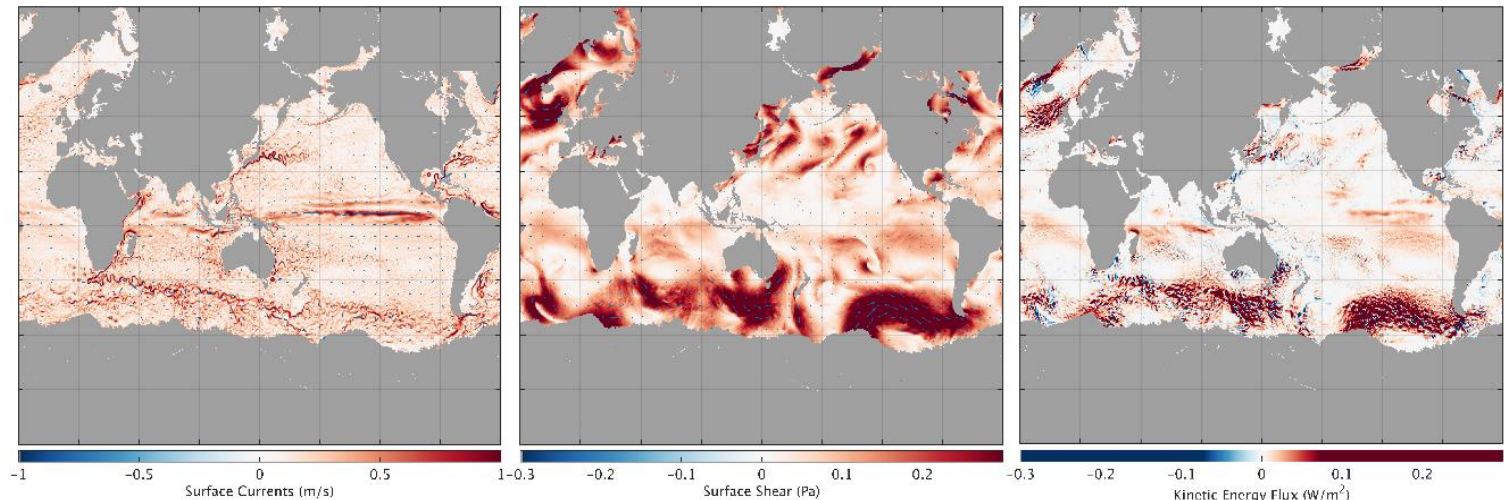

Figure 17: Left to right: Total model surface currents (7d avg), surface stress (1d avg), and KE flux computed from the previous two. Single average from Nov 2012. 
The Southern Ocean appears most distinctly positive globally, with massive patches of positive wind work due to the consistent eastern flow of the ACC matched with the large scale wind flowing in the same direction. Along the equator, the Intertropical Convergence Zone (ITCZ) provides positive work with strong currents flowing towards the west and weak winds blowing in the same direction. Looking at the mesoscale, the Gulf Stream provides an interesting look at the wind work done on the western boundary currents off the Eastern coast of the United States. The Gulf Stream is a strong positive current that flows up along the coast of the United States and eventually fans out to form the North Atlantic current. The seven day averages shown here were taken during November, at which point winds typically blow against the Gulf Stream, thus generating the strong negative wind work shown.
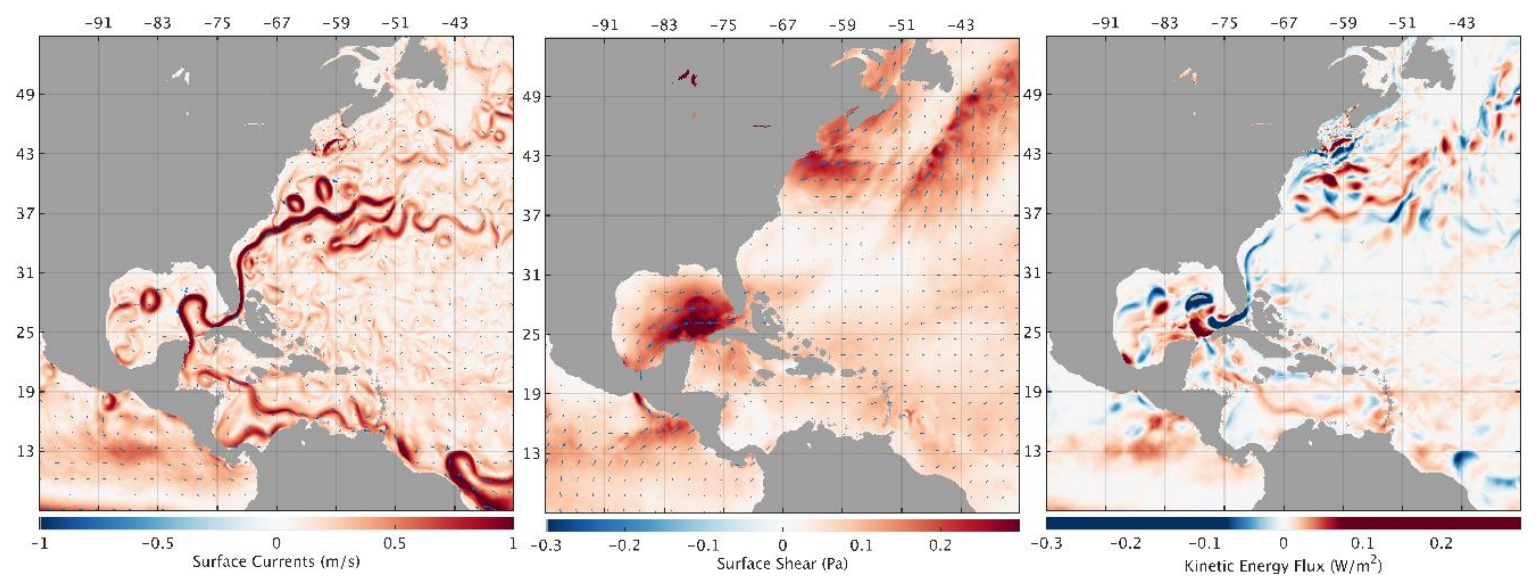

Figure 18: Zoom of Figure 17 for the Gulf Stream. Total surface currents, surface stress, and KE flux from left to right. 


\subsubsection{Geostrophic Power}

Geostrophic currents were calculated using the $1 / 48^{\text {th }}$ degree model over the same time span and averaging scheme as the total currents. In this case, sea surface height was averaged over seven days from which geostrophic surface currents and final geostrophic wind work were calculated. Since winds are taken as a 1 day average, while currents are taken as a 7 day average (here, at least), there can be some decorrelation between wind and current directions, which causes a decrease in wind work.

Figure 19 shows geostrophic surface currents, wind stress, and geostrophic KE flux from left to right. Note that a one day average of surface stress is shown since one day averages of stress were used in KE flux calculations. As expected, geostrophic currents along the equator are corrupted because geostrophy is not valid there. Similarly to the total currents above, geostrophic currents show large scale positive work in the Southern Ocean, albeit choppier than in the total currents case. Rotating eddies in the Southern Ocean cause both positive and negative work to be done when multiplied with the larger scale wind. The same ACC jets are visible, and positive work is generally done on them.
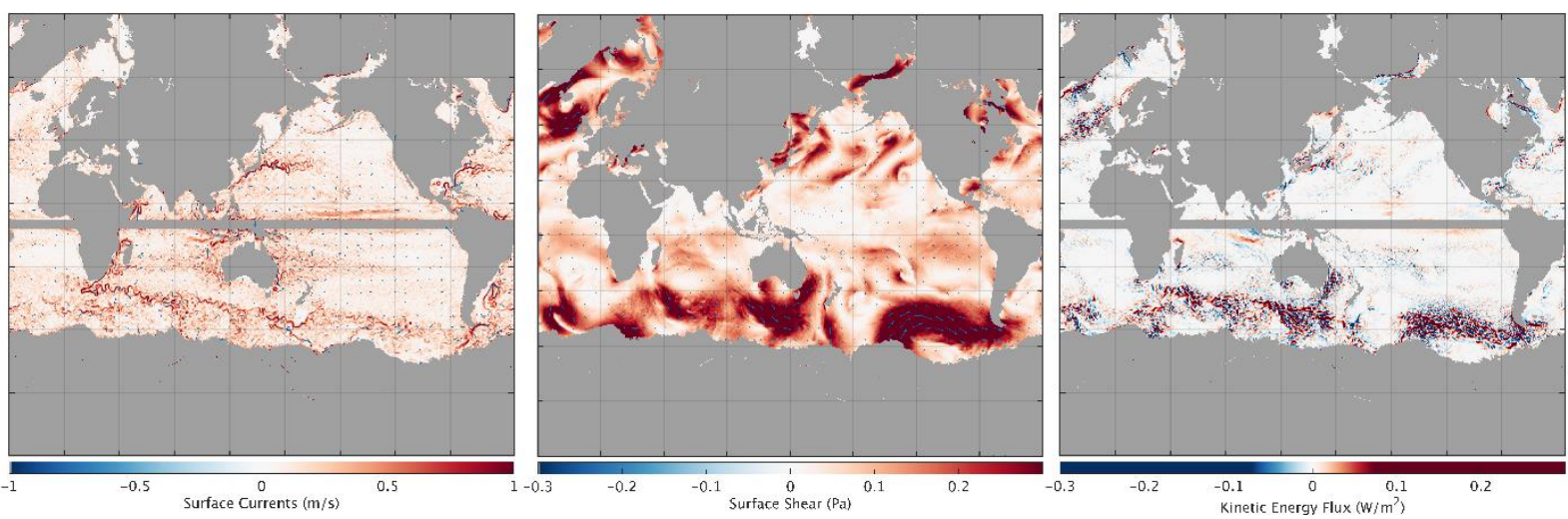

Figure 19: Left to right: Geostrophic surface currents computed from a 7d avg of sea surface height, 1d avg of surface stress, and KE flux computed from the previous two. Single averages from Nov 2012. 
Again considering the Gulf Stream as a mesoscale example, geostrophic currents predict the known strong positive flow along the eastern coast of the United States. The resulting KE flux is again strongly negative, as it was for the total currents. This is again due to strong positive currents flowing against weak wind stress. As the Gulf Stream
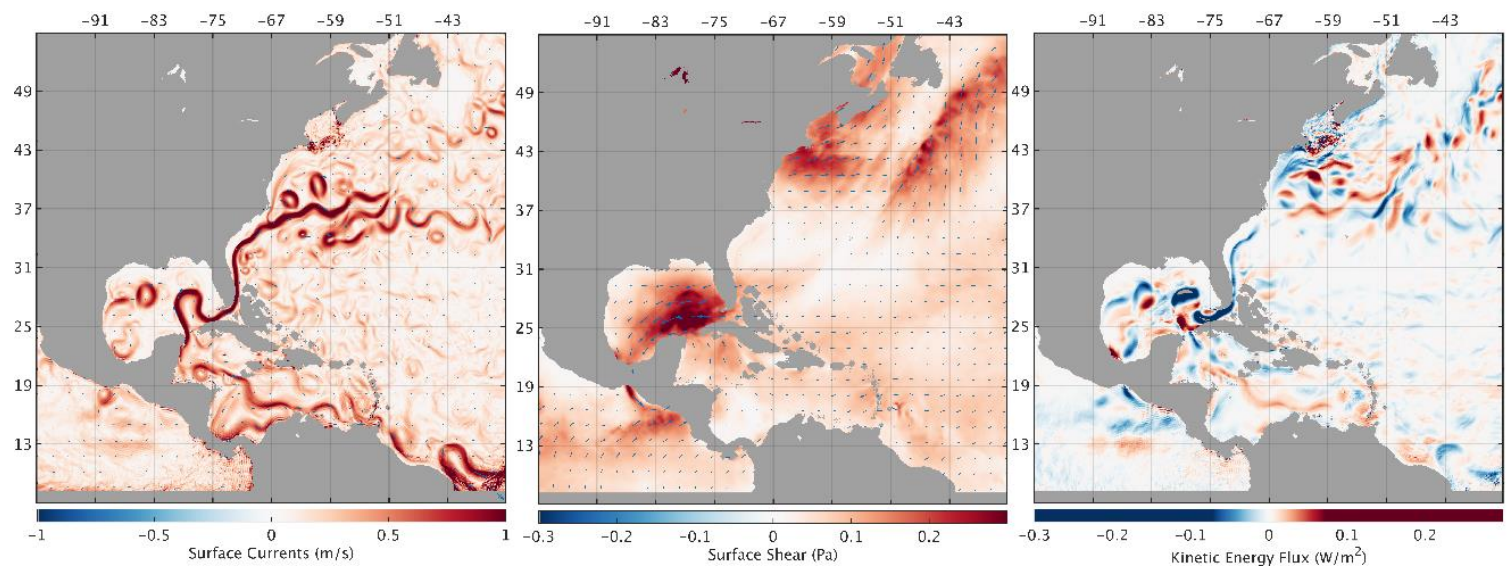

Figure 20: Zoom plot of Figure 19 for the Gulf Stream. Geostrophic currents, surface stress, and KE flux computed from the previous two from left to right.

diffuses into the North Atlantic current, both positive and negative work are done on the rotating eddies it forms. Notice the pairs of strongly negative and positive regions of wind work in the North Atlantic, which correspond to current loops in the geostrophic current figure. These are the aforementioned negative and positive work done on a rotating eddy by large scale wind.

Overall, geostrophic currents receive about $.32 \mathrm{TW}$ of power from the wind globally when a seven day average of sea surface height is used in conjunction with a one day average of surface stress. This is an order of magnitude lower than total currents, likely because geostrophic currents are not necessarily aligned with the wind stress, meaning both positive and negative wind work is done on rotating eddies. 


\subsubsection{Ekman Power}

As with the two previous current types, Ekman currents are shown as seven day averages and were calculated as specified in the Analysis section. Since simple Ekman dynamics were used in these calculations, resulting currents will always be rotated 45 degrees to the wind vector. For this reason, Ekman generated wind work should always be positive - though this won't necessarily be the case here due to wind-current decorrelation caused by averaging.

Figure 21 shows the calculated seven day Ekman currents, one day surface stress, and corresponding KE flux. The majority of the ocean is positive, with small patches of slightly negative KE flux due to averaging decorrelation. The large scale wind patterns essentially translate into large scale wind work, particularly in the southern ocean where the winds are consistent in direction and fairly strong in magnitude. In areas of less consistent wind, averaging decorrelation reduces power input. Areas with weaker wind stress also produce lower power, since Ekman currents are directly determined by wind stress.
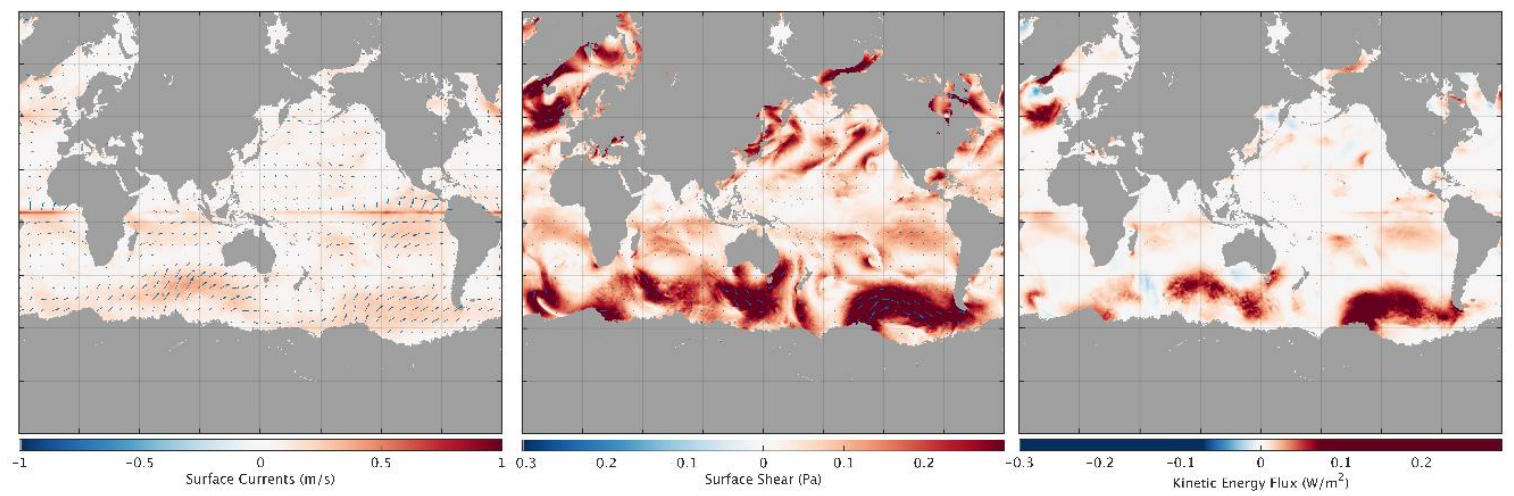

Figure 21: Surface Ekman currents, surface stress, and kinetic energy flux computed from the previous two. 
The Gulf Stream is less interesting here than it was when looking at geostrophic currents. Since the Gulf Stream is primarily composed of geostrophic currents, not much shows up in terms of Ekman currents. The relatively calm winds produce little in the way of currents, and the resulting wind work is similarly small.
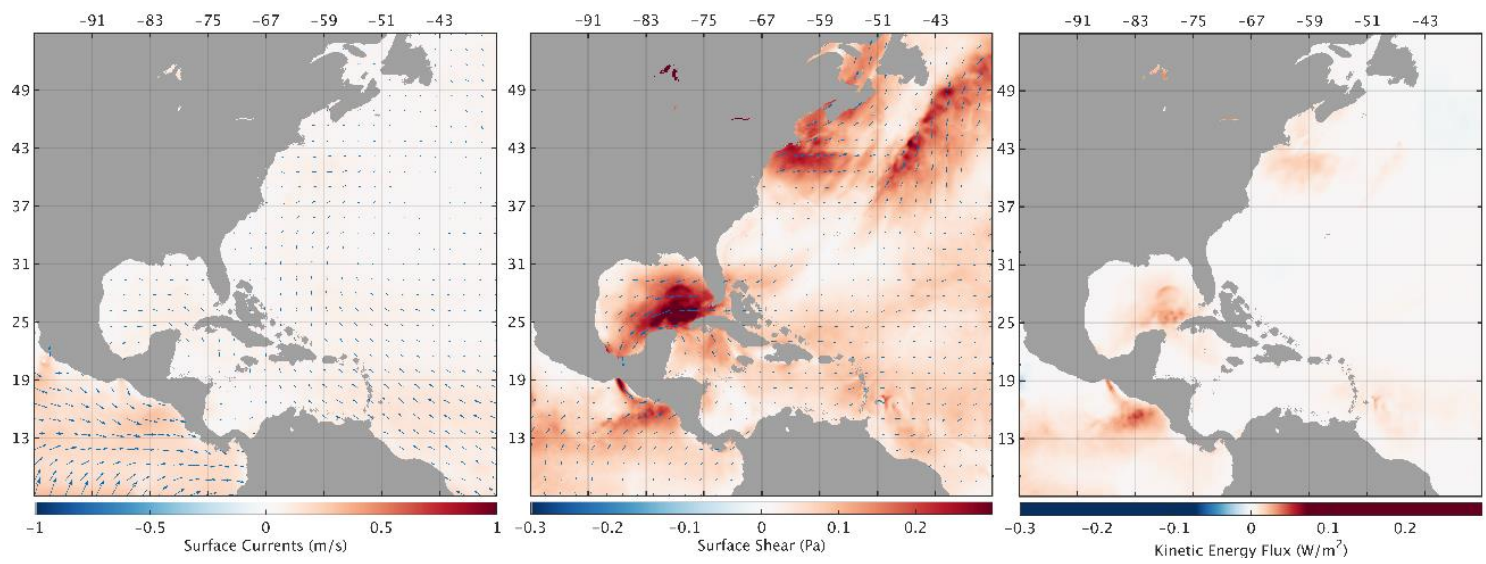

Figure 22: Zoom plot of Figure 21 for the Gulf Stream. From left to right: Ekman currents, surface stress, and KE flux computed from the previous two.

Globally, Ekman currents receive about 3.05 TW of power from the wind when computed from seven day average currents and one day average wind stress in the $1 / 48^{\text {th }}$ degree model.

\subsubsection{Ageostrophic Power}

Ageostrophic currents are approximated as Ekman currents in the majority of this work (and other wind power literature). This is because Ageostrophic currents are difficult to calculate directly - they are made up of many difficult to model currents. Thus, the question of how good the Ekman current approximation is presents itself.

Since ageostrophic currents are the currents that are not in geostrophic balance, subtracting geostrophic currents from total currents should be another way of calculating 
ageostrophic currents. This was done with the $1 / 48^{\text {th }}$ degree model data, with an average of all seven day averages between September 2011 and February 2012. The resulting spatial maps are shown in Figure 23. There is very little difference between the two maps, showing how good the Ekman approximation is on average.
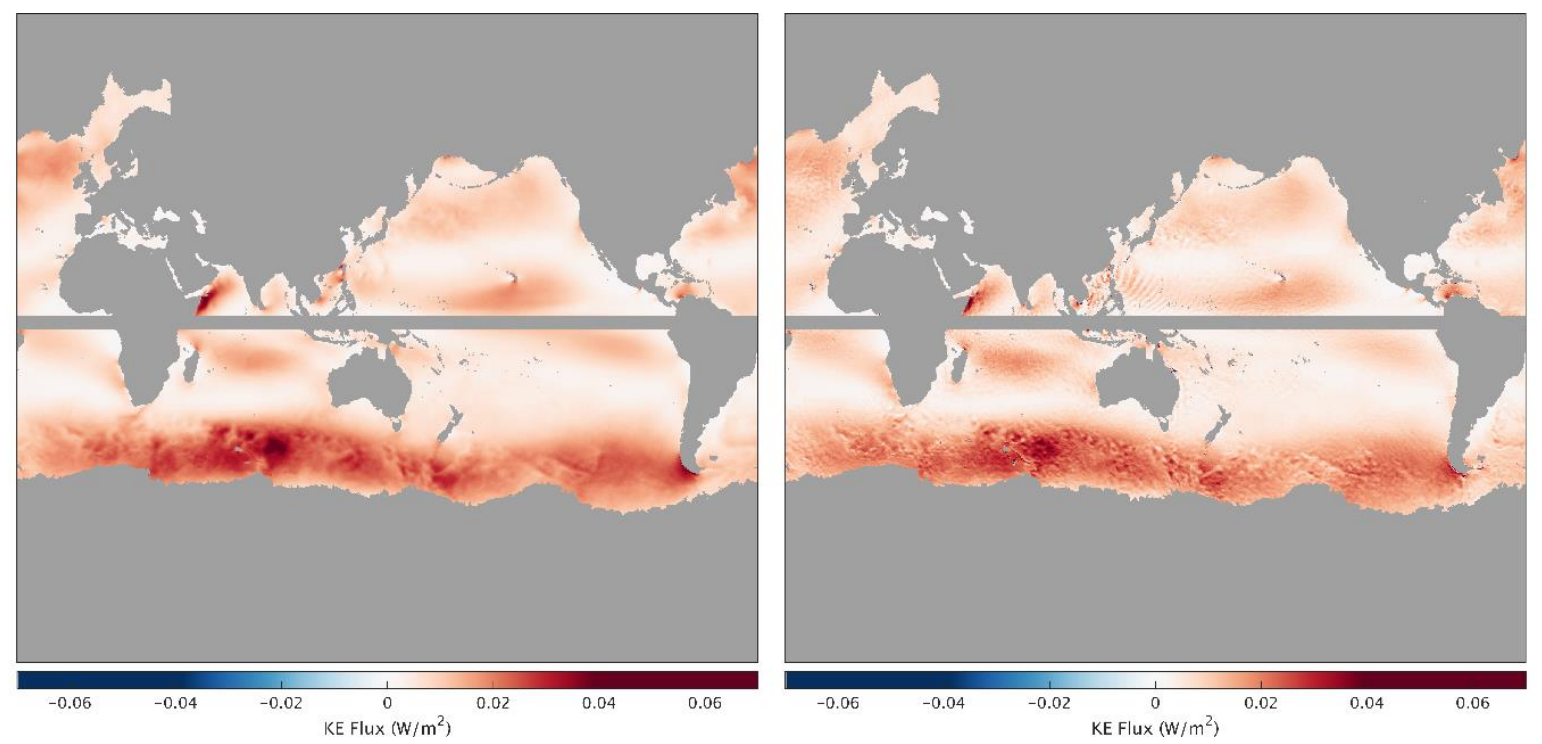

Figure 23: Comparison of KE flux for Ekman currents (left) and Ageostrophic currents (right).

\subsubsection{Zonal Averages}

While interesting, the above spatial plots do little to put numbers to wind work. Figure 24 shows zonally (latitude bands) averaged kinetic energy flux for the different current types. For all the currents analyzed, the Southern Ocean shows a large spike in KE flux, with a similarly large spike for the wind generated currents near 60 degrees north. As in the spatial maps, the zonal averages show geostrophic and Ekman currents 
summing to the total currents as hoped - another indication that the Ekman approximation works well on average.

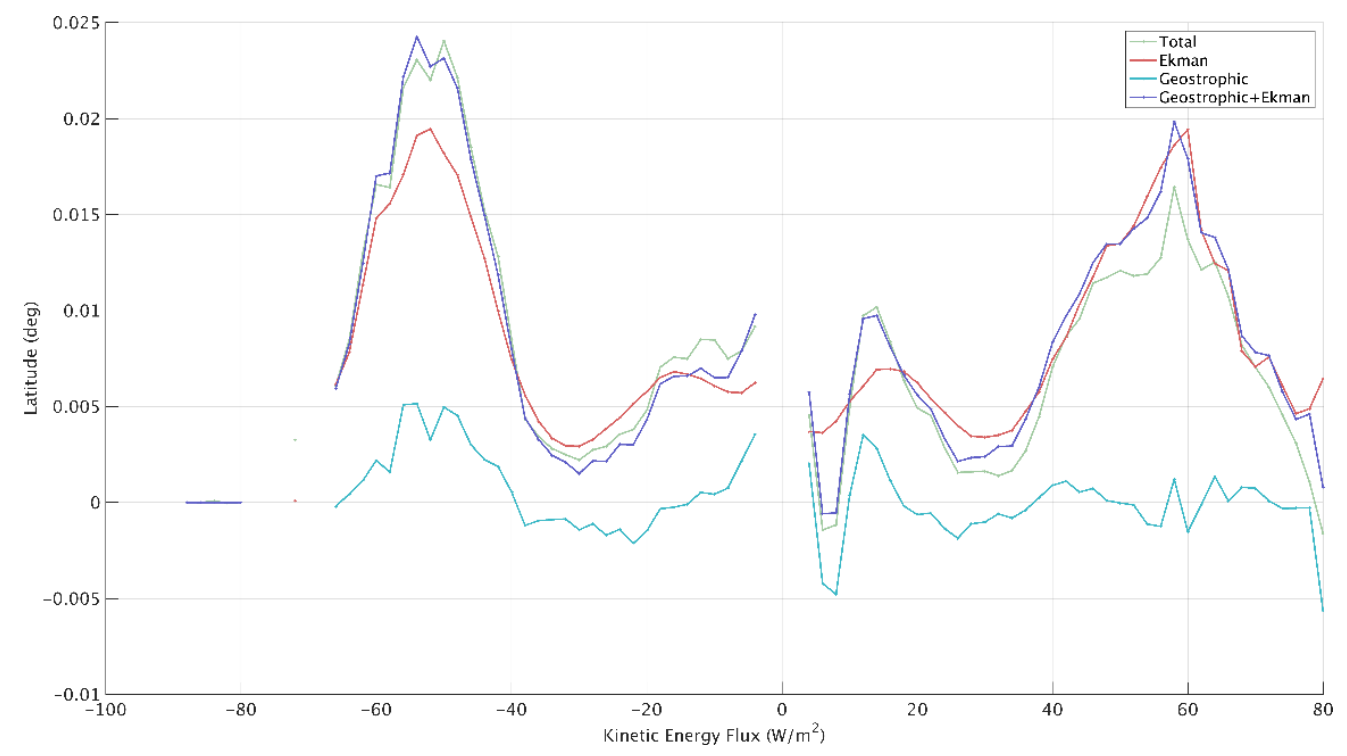

Figure 24: Zonal averages of kinetic energy flux for total model, Ekman, geostrophic, and the total approximation geostrophic+Ekman.

\subsection{Time Sensitivity}

There are two time factors at play here. First: currents, wind, and thus wind work vary on a broad range of time scales. This wind work variability is only briefly touched on here. The second factor, averaging period, is much more heavily investigated.

\subsubsection{Wind Work Time Series}

Since the $1 / 48^{\text {th }}$ degree model only spans a few months, the $1 / 24^{\text {th }}$ degree model was used for time series plotting. The $1 / 24^{\text {th }}$ degree model data used covers the time period from January 2012 through April 2013. Within the 15 month period analyzed, a few breaks occur due to missing or corrupted data. Figure 26 plots windowed, seven day 
averages for total, Ekman, and geostrophic wind work. While interesting, the time series plot does not show a lot of useful information on account of the relatively short time span. More information can be gleaned from taking seasonal averages of the time series data, as found in Figure 27.

Figure 27 shows the Northern Hemisphere seasonal averages for one year of $1 / 24^{\text {th }}$ degree data. Error bars represent the standard error of the mean for each group. Since seven day averages are computed daily, there may be some correlation between adjacent daily samples, which could cause an underestimation of standard error (autocorrelation). Still, there does appear to be a substantial difference between seasons. Total currents (MITgcm) result in 3.49 TW of wind work during the summer, as opposed to just 2.84 TW during the fall. Similarly, geostrophic currents see about .4 TW of wind work during the summer and just .29 TW during the fall. Interestingly, Ekman currents see very little variation in wind work. The increases in wind work for geostrophic and total currents during the summer are likely due to the Southern Ocean winter when the ACC intensifies. 


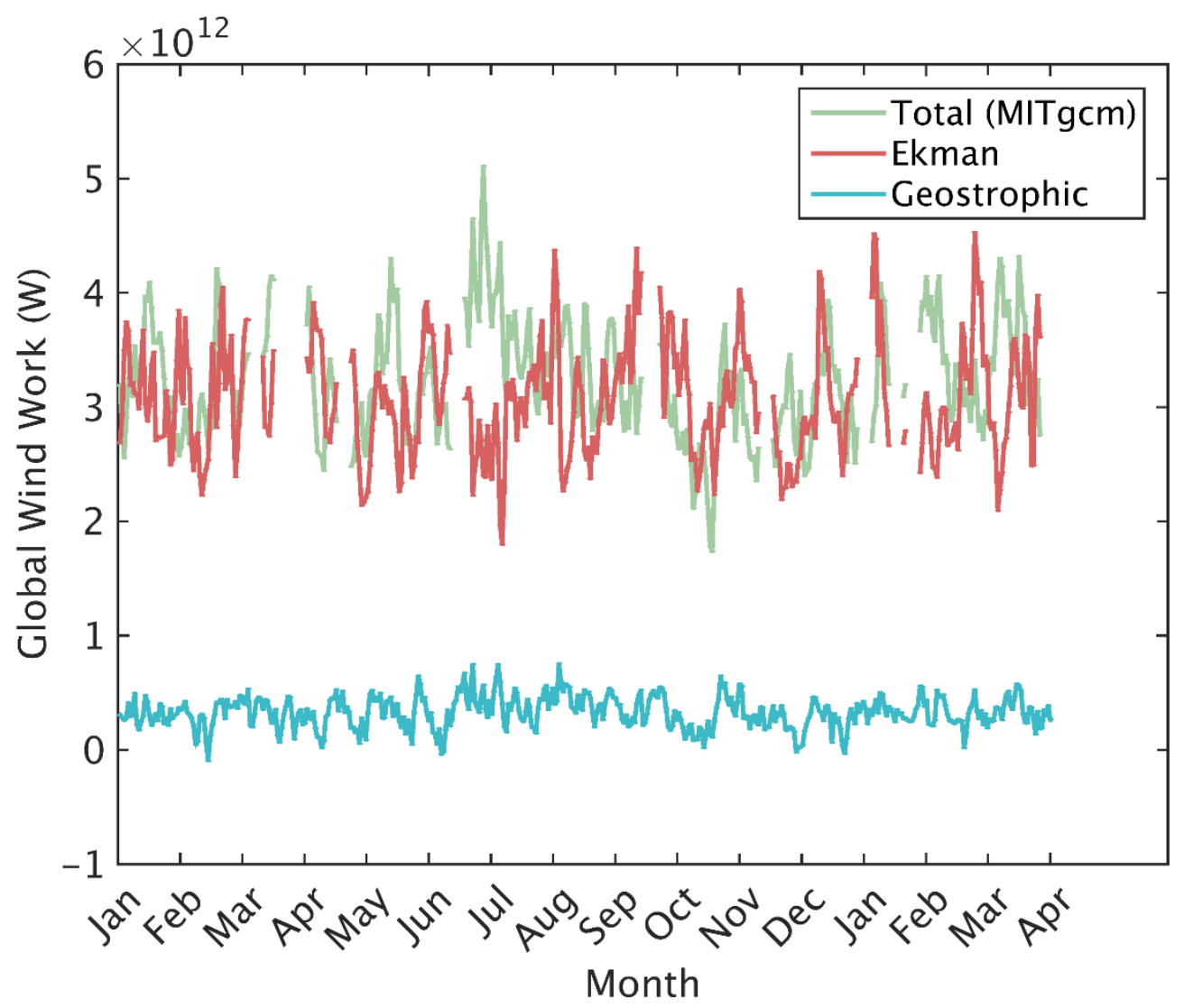

Figure 25: Time series for total power over 15 months on $1 / 24^{\text {th }}$ degree data. Seven day window averaging used for currents, one day for surface stress.

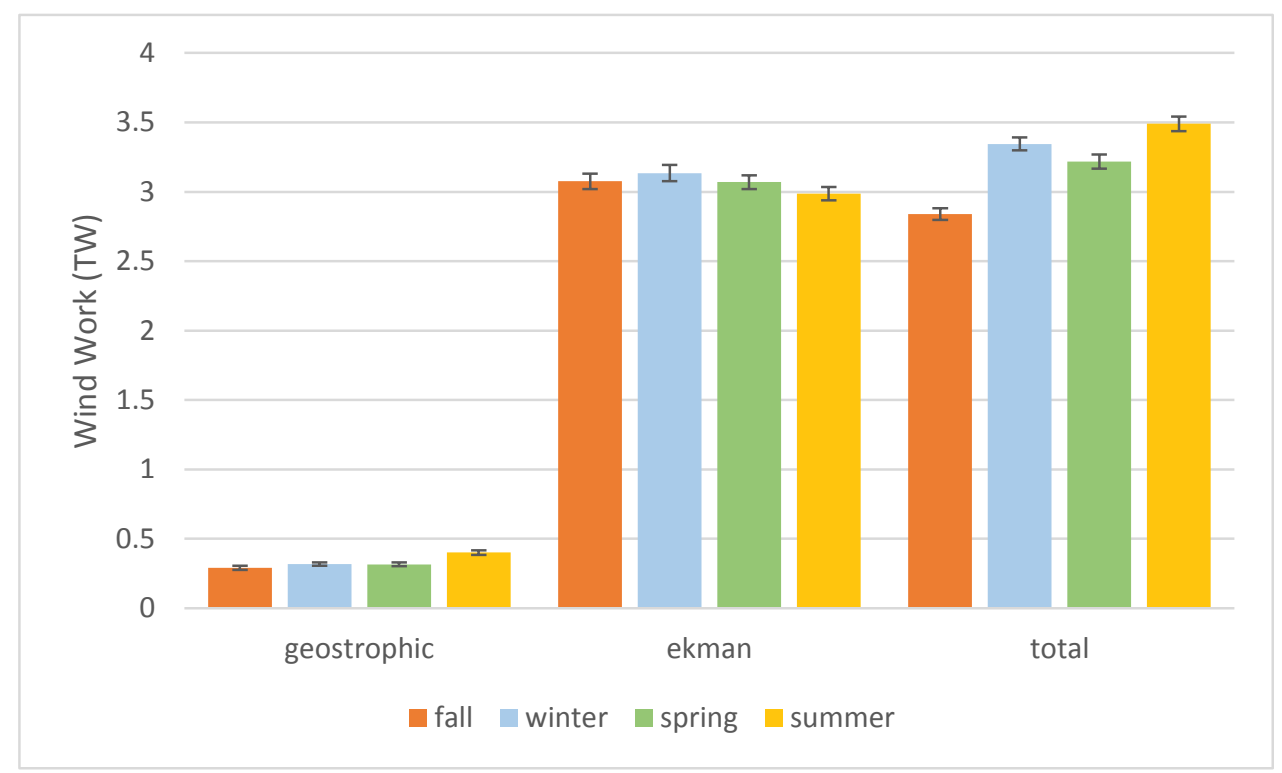

Figure 26: Seasonal averages for one year of $1 / 24^{\text {th }}$ degree data. Seven day window averaging for currents, one day for surface stress. Standard error bars are optimistic due to autocorrelation. 


\subsubsection{Averaging Period}

To determine the effect of averaging period on global wind work, Ekman, geostrophic, and total currents were calculated using data of varied averaging periods. One, three, seven, eleven, and fifteen day averages of currents were considered with a constant one day average of wind stress in each case.

Figure 27 shows the globally integrated wind work for each current type over averaging period. Ekman currents show a negative trend with averaging period, which can be explained by the decorrelation of wind and wind-driven currents as averaging period increases. A similar argument can be made for total currents, since they are primarily composed of ageostrophic (Ekman) currents. Since geostrophic currents are not wind driven, they do not experience the same decorrelation as averaging period increases.

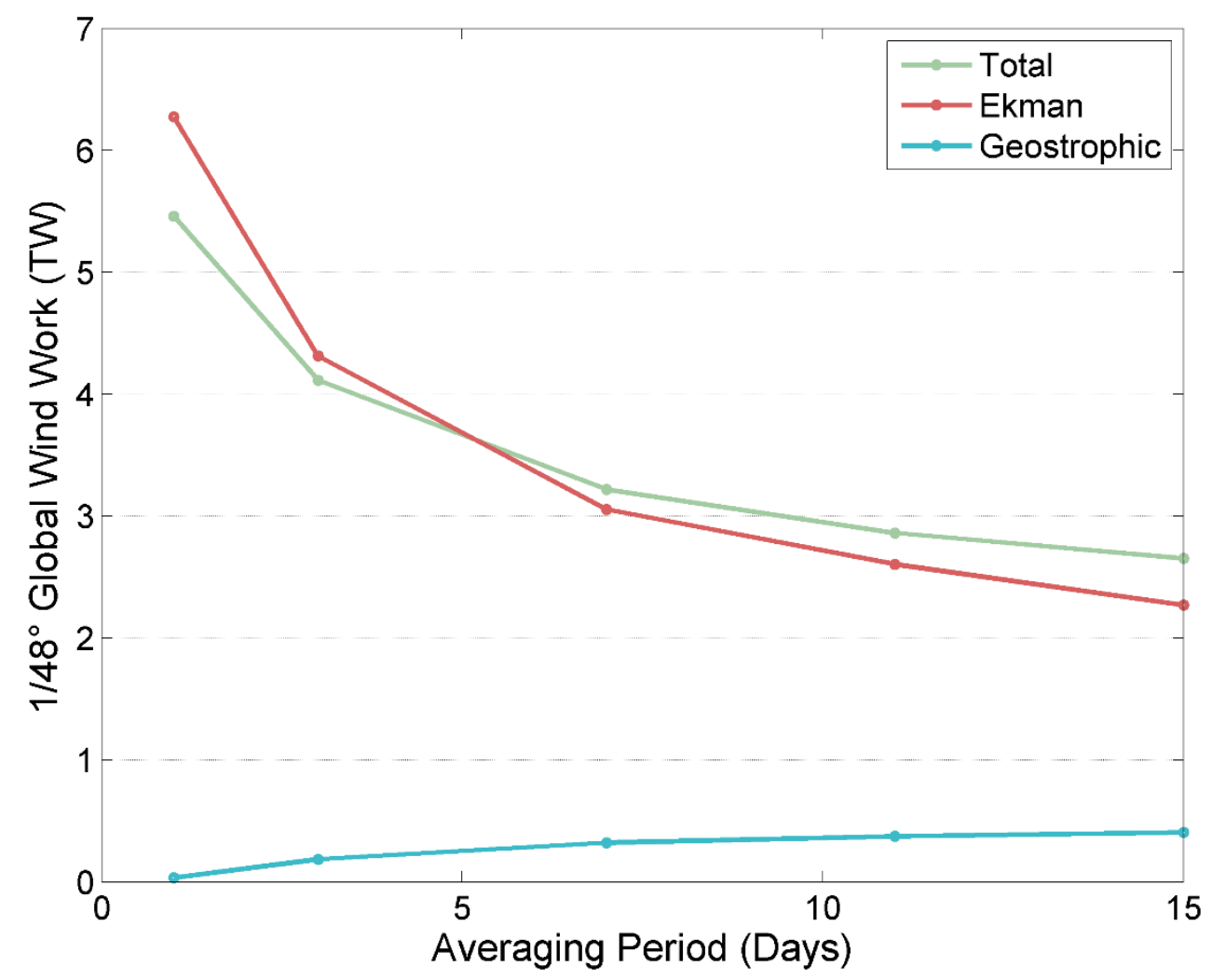

Figure 27: The effect of averaging period on wind work. Data taken from $1 / 48^{\text {th }}$ degree model. 
Indeed, the opposite trend occurs. It seems that averaging over longer time periods results in a larger calculated geostrophic wind work. This is a significant result, as it implies that the instantaneous geostrophic wind work is nearly zero, likely because positive and negative regions of geostrophic wind work cancel each other out.

The crossover between total and Ekman currents is an interesting phenomena. This is the point at which geostrophic currents start to become a significant factor in total currents - at about five days. If a single averaging period is to be selected, this point should be taken into account since an ideal wind work measurement would not overdecorrelate wind and Ekman currents, but would also give geostrophic currents enough time to develop. To be near this point, but close to where the asymptotes level out, a seven day average was chosen for single period calculations (this is the reason for seven day averages in the preceding sections).

Figures 28, 29, and, 30, show the total, geostrophic, and Ekman zonal averages of kinetic energy flux as a factor of averaging period. These calculations were all made on the $1 / 48^{\text {th }}$ degree model, which means data is from northern hemisphere fall and winter months (SONDJ). With the seasons in mind, the Southern Ocean expectedly shows very high wind work over all averaging periods. Interestingly, the difference between one day and fifteen day averages is significantly more pronounced in the Northern Hemisphere higher latitudes than in the Southern Hemisphere. This is likely due to the Southern Ocean ACC, which is generally aligned with the wind. As averaging period increases, the mean ACC is still well aligned with the mean winds, so less decorrelation takes place. Conversely, in the Northern Hemisphere, where western boundary currents, the North Atlantic, and North Pacific currents generate significant power, wind-current 
decorrelation plays a larger factor. Geostrophic currents show a similar, but much less pronounced trend, with larger differences in KE flux at more extreme latitudes - near the equator there is no discernable difference.

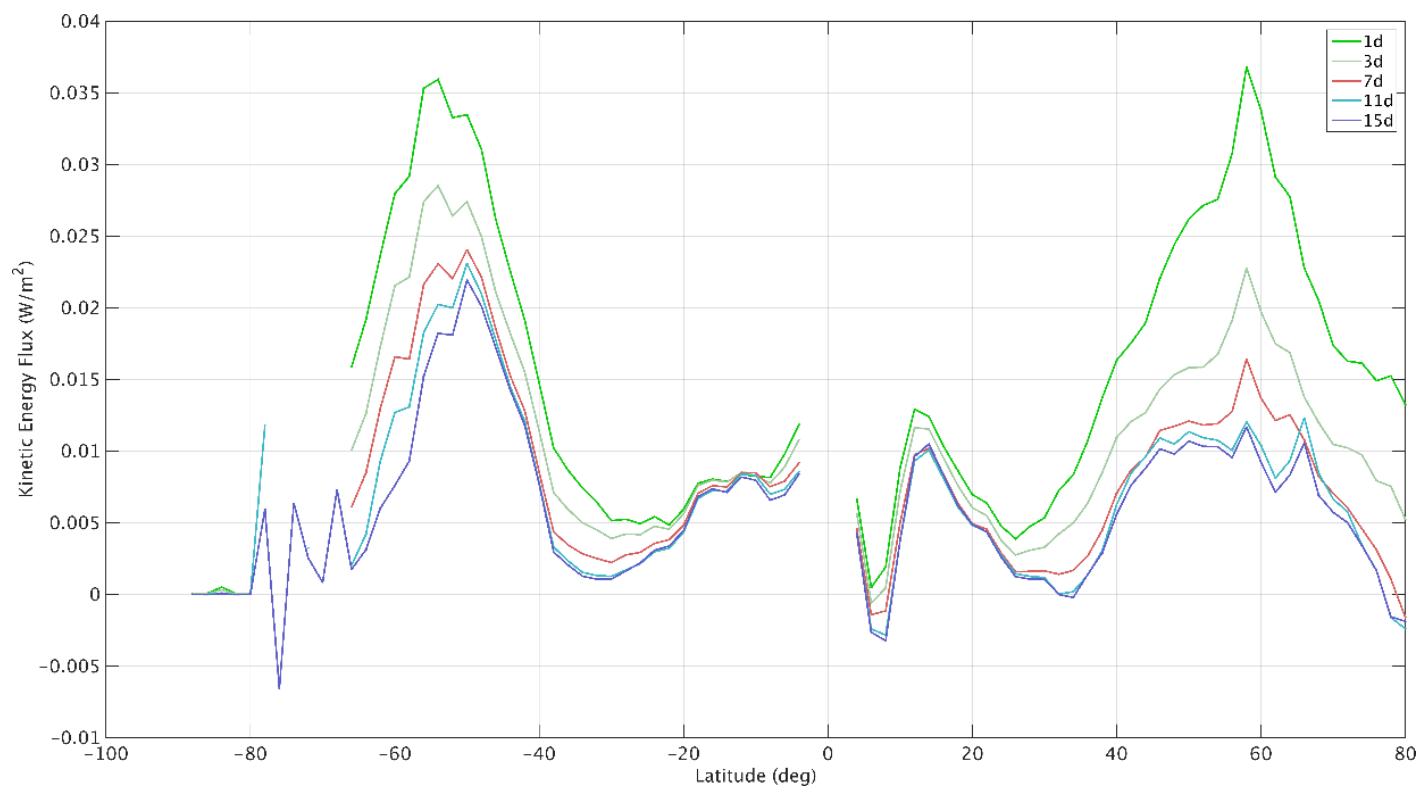

Figure 28: Zonal averages of KE flux for total currents over multiple current averaging periods. Surface stress averaging held constant at one day. 


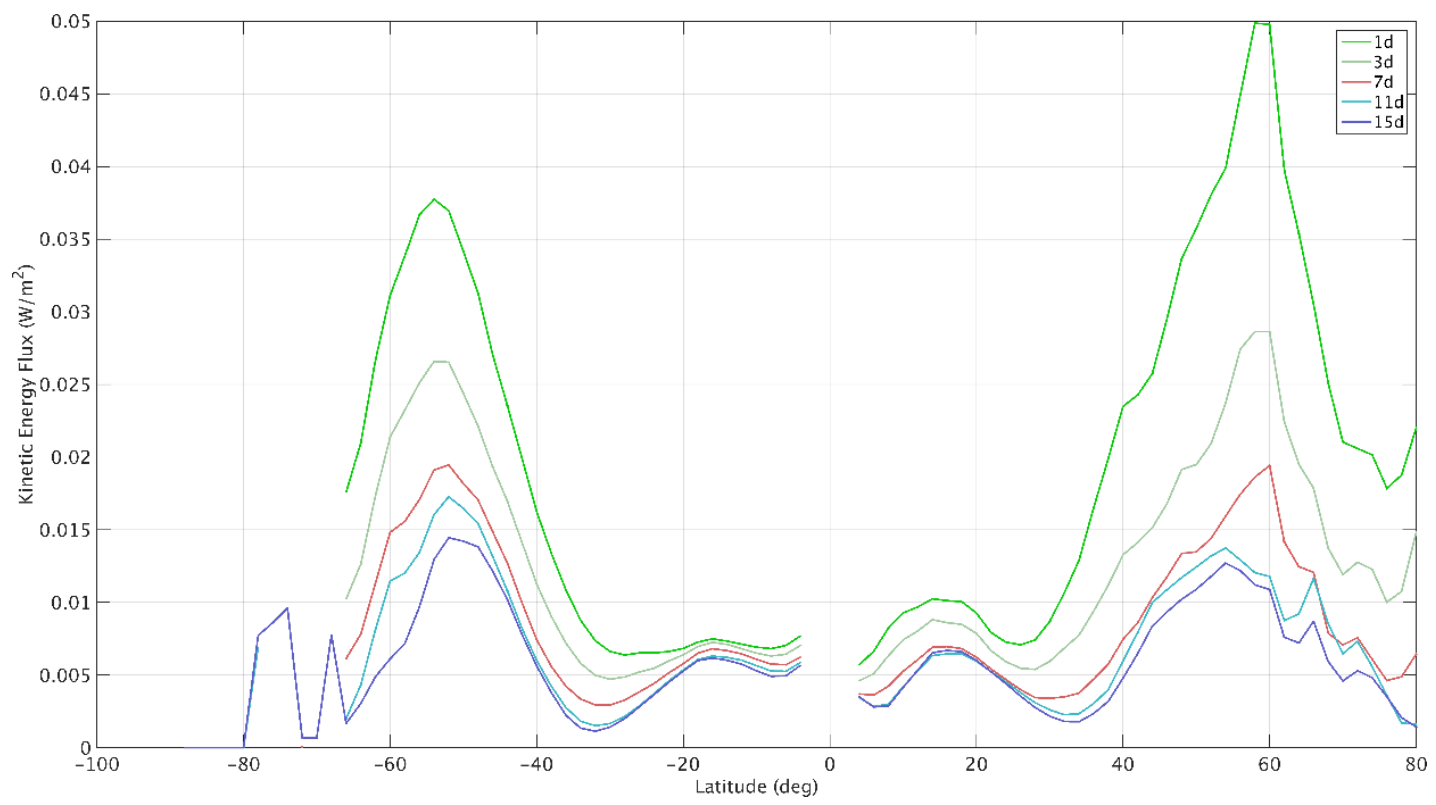

Figure 29: Zonal averages of KE flux for Ekman currents over multiple current averaging periods. Surface stress averaging held constant at one day.

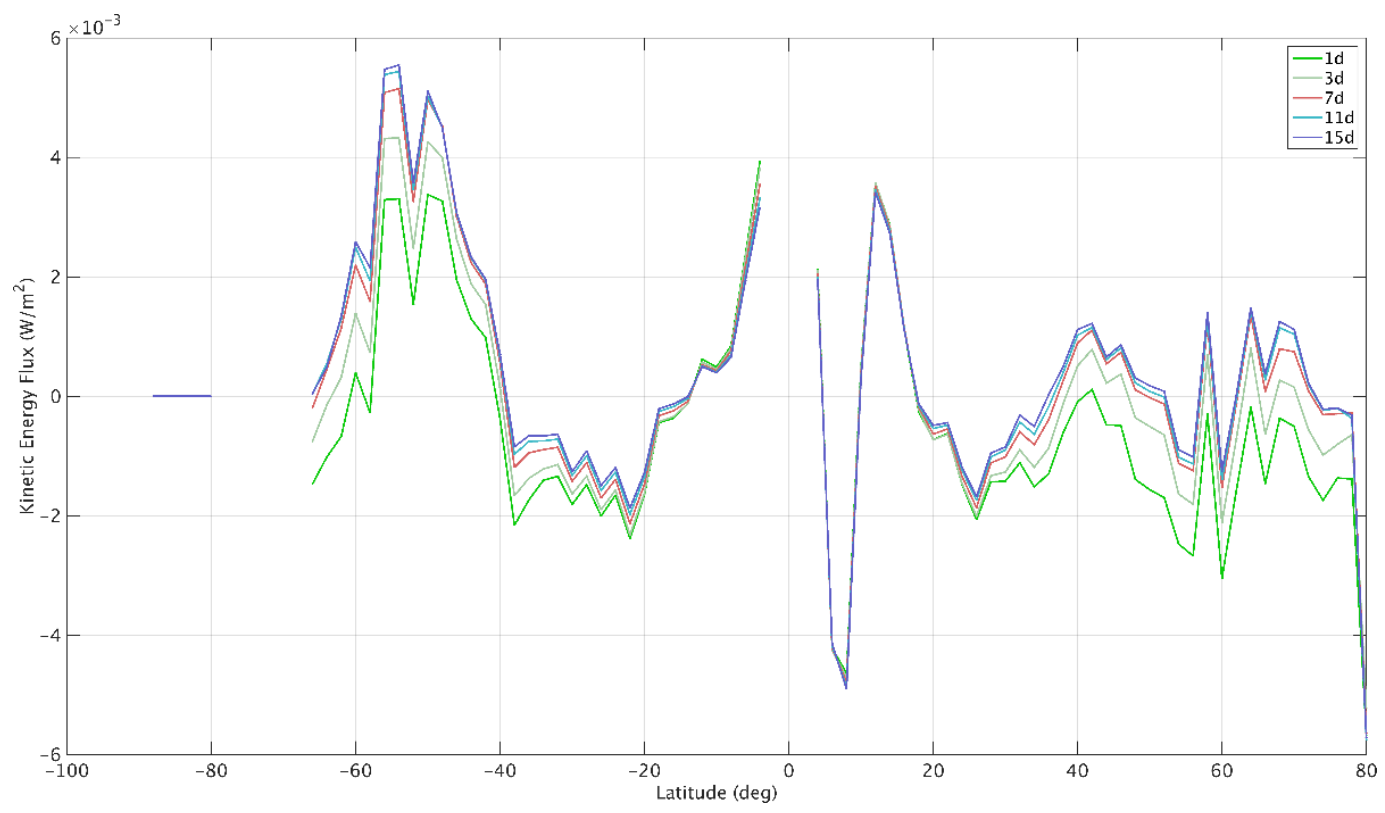

Figure 30: Zonal averages of KE flux for geostrophic currents over multiple current averaging periods. Surface stress averaging held constant at one day. 


\subsection{Spatial Resolution Effects}

Two spatial resolutions were considered in this work: $1 / 24^{\text {th }}$ degree and $1 / 48^{\text {th }}$ degree models. Both of these models should be capable of resolving mesoscale processes, and the $1 / 48^{\text {th }}$ degree model begins to resolve sub-mesoscale processes with its better than $2 \mathrm{~km}$ nominal resolution. Total $(\mathrm{MITg} \mathrm{cm})$ global wind work was calculated for each model at multiple averaging periods; $1,3,7,11$, and 15 days. Since the $1 / 48^{\text {th }}$ degree model only spans the months of September through February 2012/2013, the $1 / 24^{\text {th }}$ degree model was subsampled to match the same time period.

Figure 31 plots the effect of resolution over averaging period. The $1 / 48^{\text {th }}$ degree model shows slightly lower wind work at the lower averaging time spans. Standard errors appear to show a significant difference at 1 sigma, but caution should be used since there

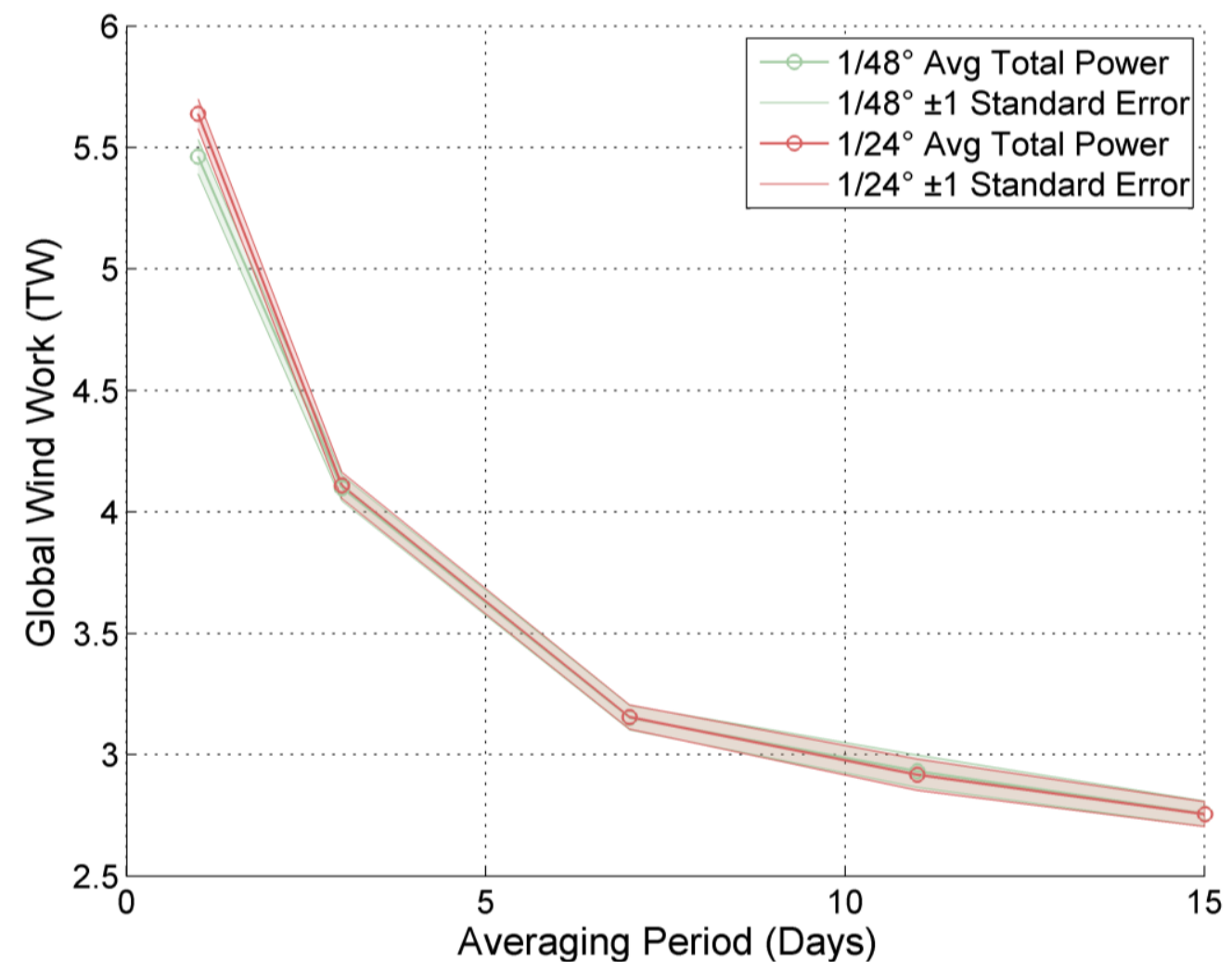

Figure 31: Averaging period and resolution effects on global wind work. 
is likely autocorrelation in the data and errors. In theory, the lower wind work in the $1 / 48^{\text {th }}$ degree model does make sense, though, since the sub-mesoscale processes visible in the higher resolution model are likely not correlated with the large scale wind, and thus produce negative wind work.

This decrease in wind work only shows up at the lower averaging periods likely because small scale processes average out over a relatively short time span, meaning the negative wind work will only show up at the short term averaging periods. This is an interesting, and theoretically sound result, but no firm judgement is made here on whether it is significant. More data is needed (longer time period) to make a statistically sound conclusion.

Looking at the spatial difference maps, it appears that the majority of the differences are in regions of markedly geostrophic currents. This could indicate that the resolution differences occur mainly in the geostrophic case, and that the total currents are masking the effect. Further analysis, however, is left to another study.
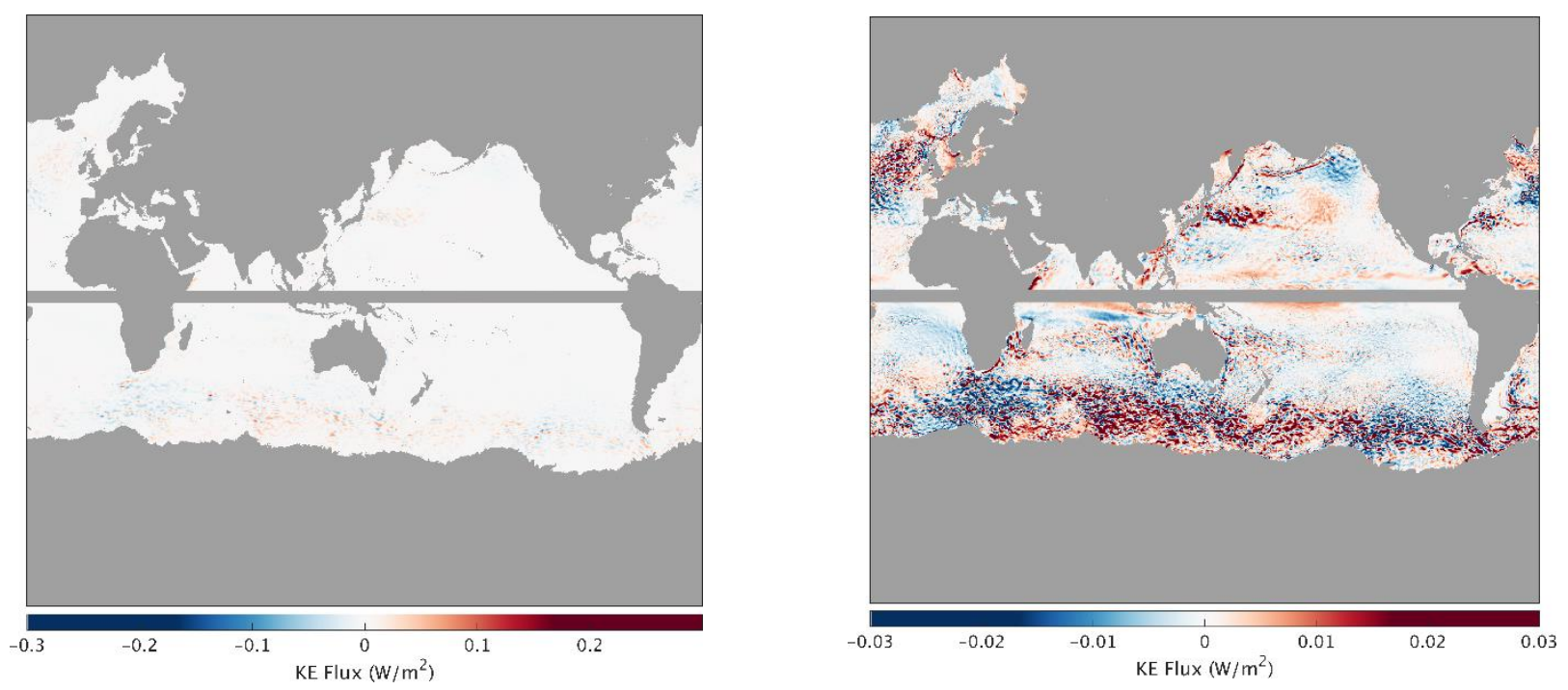

Figure 32: Spatial maps of $1 / 24^{\text {th }}$ degree total power minus $1 / 48^{\text {th }}$ degree total power for a 7 day average. Both maps are the same, but with different color scales. 


\subsection{DopplerScat Simulation}

For simulated DopplerScat observations, daily snapshots of model data were filtered with a variable length Gaussian filter, down sampled, noised, and finally averaged before used to compute wind work. This process was completed for 8 days in November of 2011. Figure 33 shows the ideal power input for the 8 days selected, simply

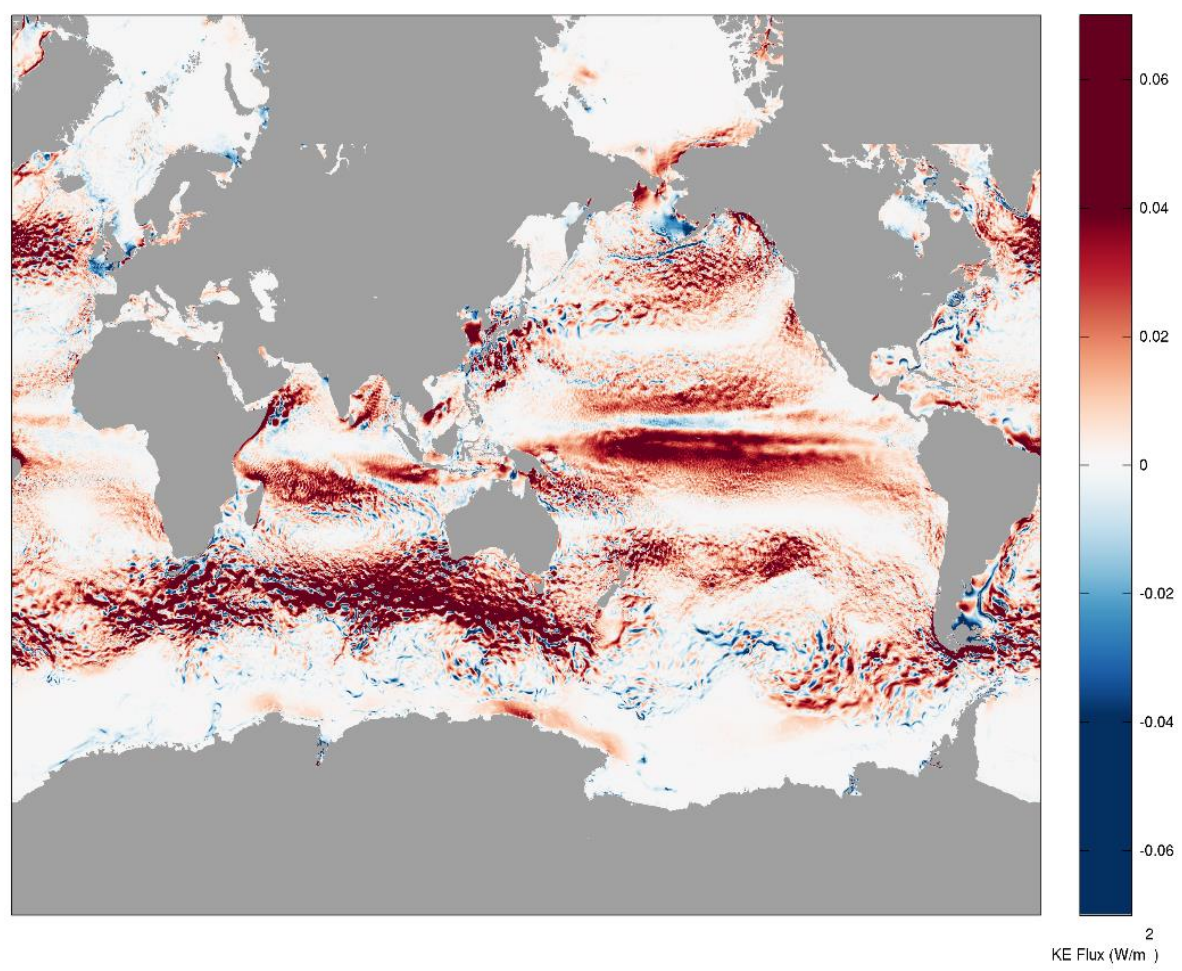

Figure 33: No noise, filtered and downsampled total currents KE flux.

down sampled from the model data. With no noise, the total nominal power input for the down sampled data is $3.66 \mathrm{TW}$. This is in line with what would be expected when comparing to the previous section's results. The noisy (Figure 35) and no-noise (Figure 31) spatial maps look similar, with the strong wind work sources appearing in both.

While some of the weaker sources are more difficult to discern in the noisy version, even weak sources are still visible. The main effect of the noise appears to be the 
spreading out of features. In the Southern Ocean, for example, where there is typically strong positive wind work, noise causes the previous concentrated cells to spread out. This difference does not appear to substantially affect the overall power levels, however, with 3.60 TW and 3.66 TW generated in the no noise and noisy cases respectively. To help bring the noisy spatial map closer to the original version, a $3 \times 3$ median filter was used to de-noise the noisy result. This is shown in Figure 34, where much of the aforementioned noise spread has been removed. While sub-mesoscale features were never on the table (the downsampled resolution is too large for sub-mesoscale features to be visible, regardless of noise), mesoscale features are certainly visible in the median and even in some places in the noisy figure.

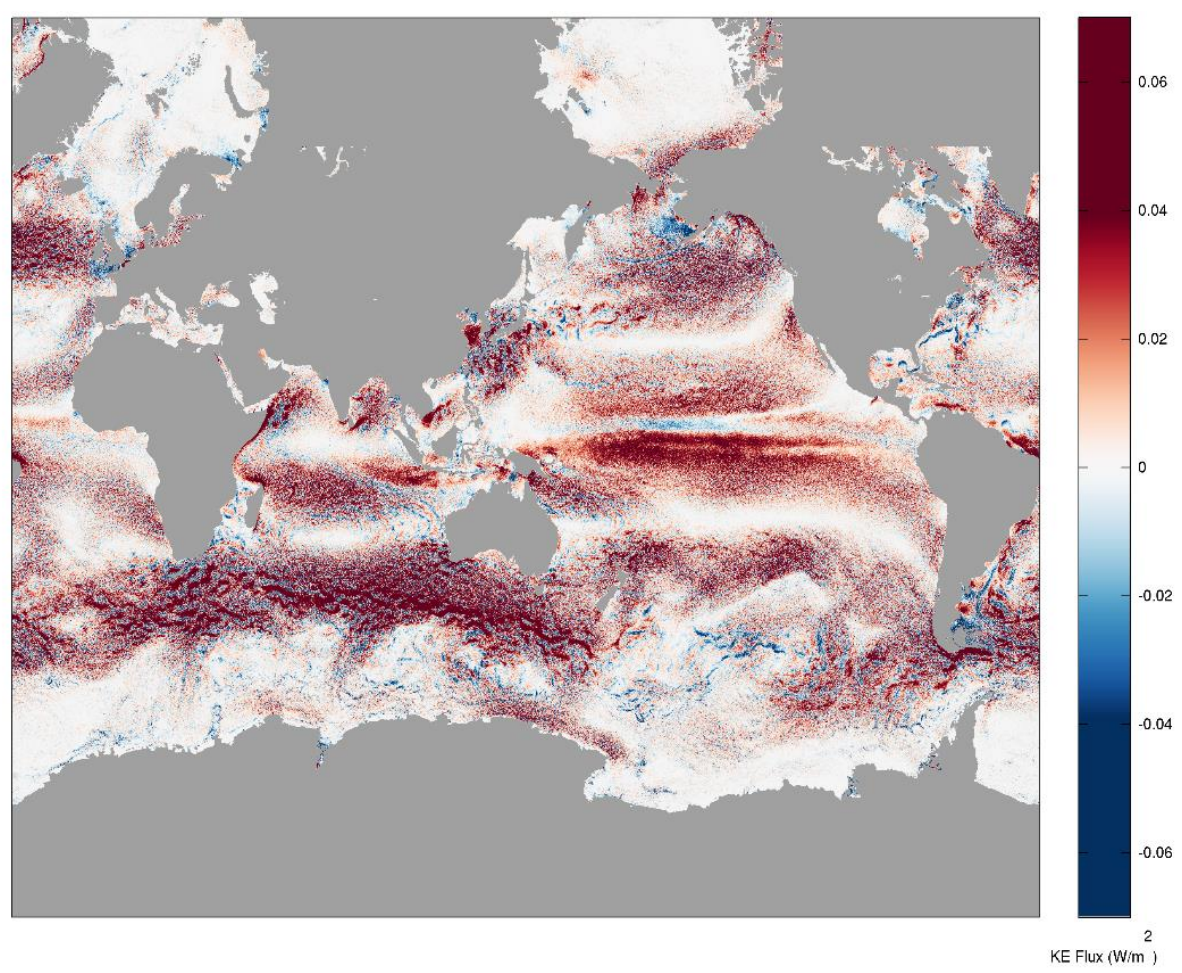

Figure 34: Filtered, downsampled, noisy KE flux computed from total currents. 


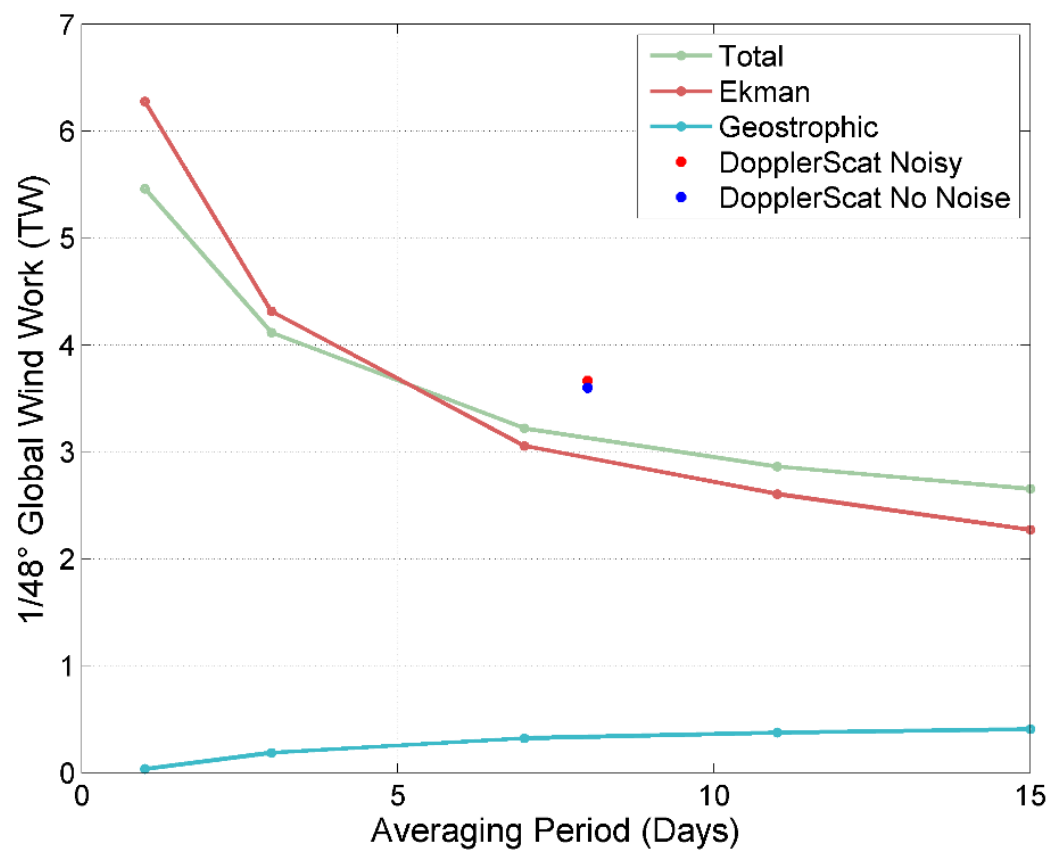

Figure 35: Comparison of DopplerScat noisy KE flux to $1 / 48^{\text {th }}$ degree model data.
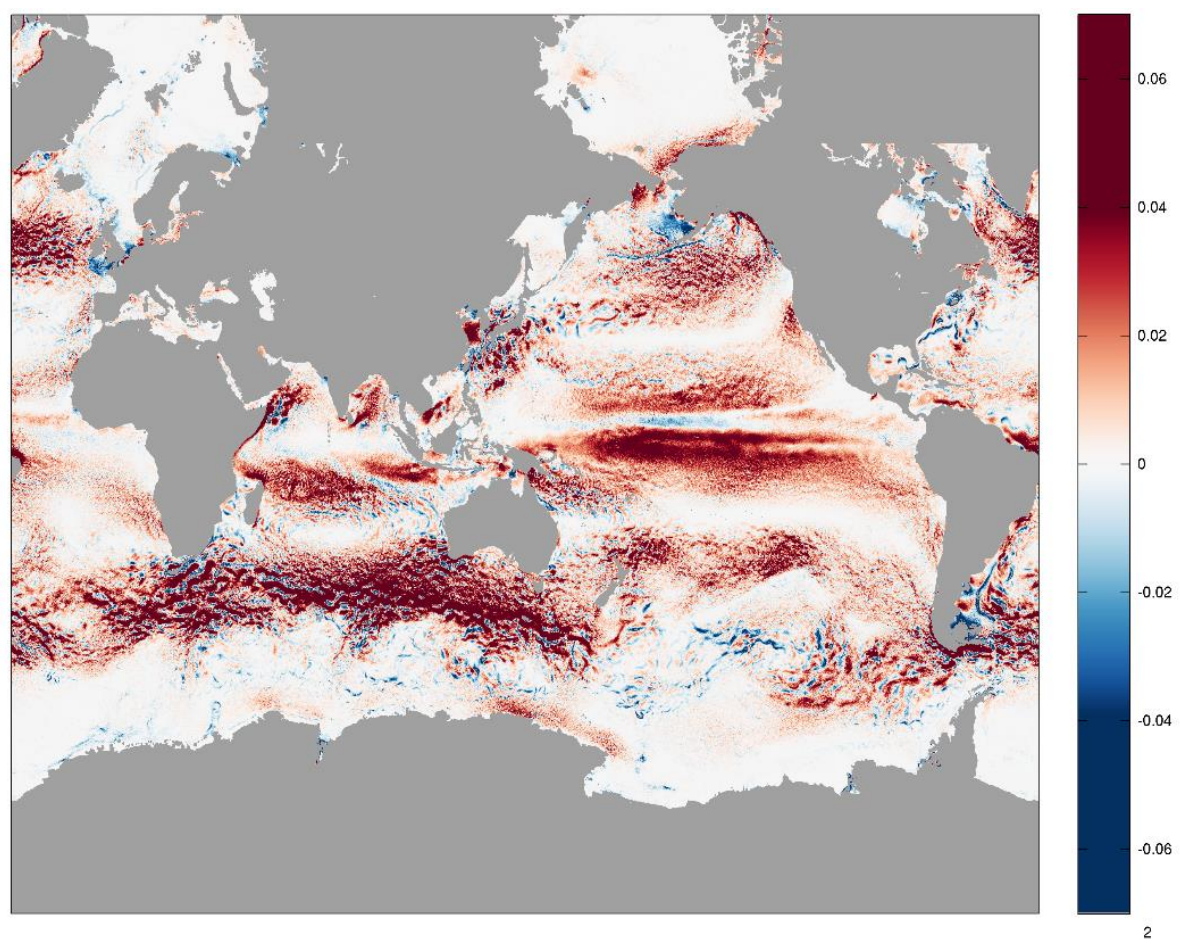

Figure 36: Median filtered version of Figure 34. 
The strongly negative Gulf Stream is clearly visible in both the noisy and median filtered figures, as is the weakly negative Equitorial band near 10 degrees North. The noisy eight day average offers a surprisingly large range of KE flux detection.

The zonal averages shown in Figure 37 reiterate what the global average and spatial maps already show. There is extremely good agreement between the noisy and no noise wind work calculations. At worst, there is a marginal difference near the equator. This means that DopplerScat should have no problems with any particular zonal region.

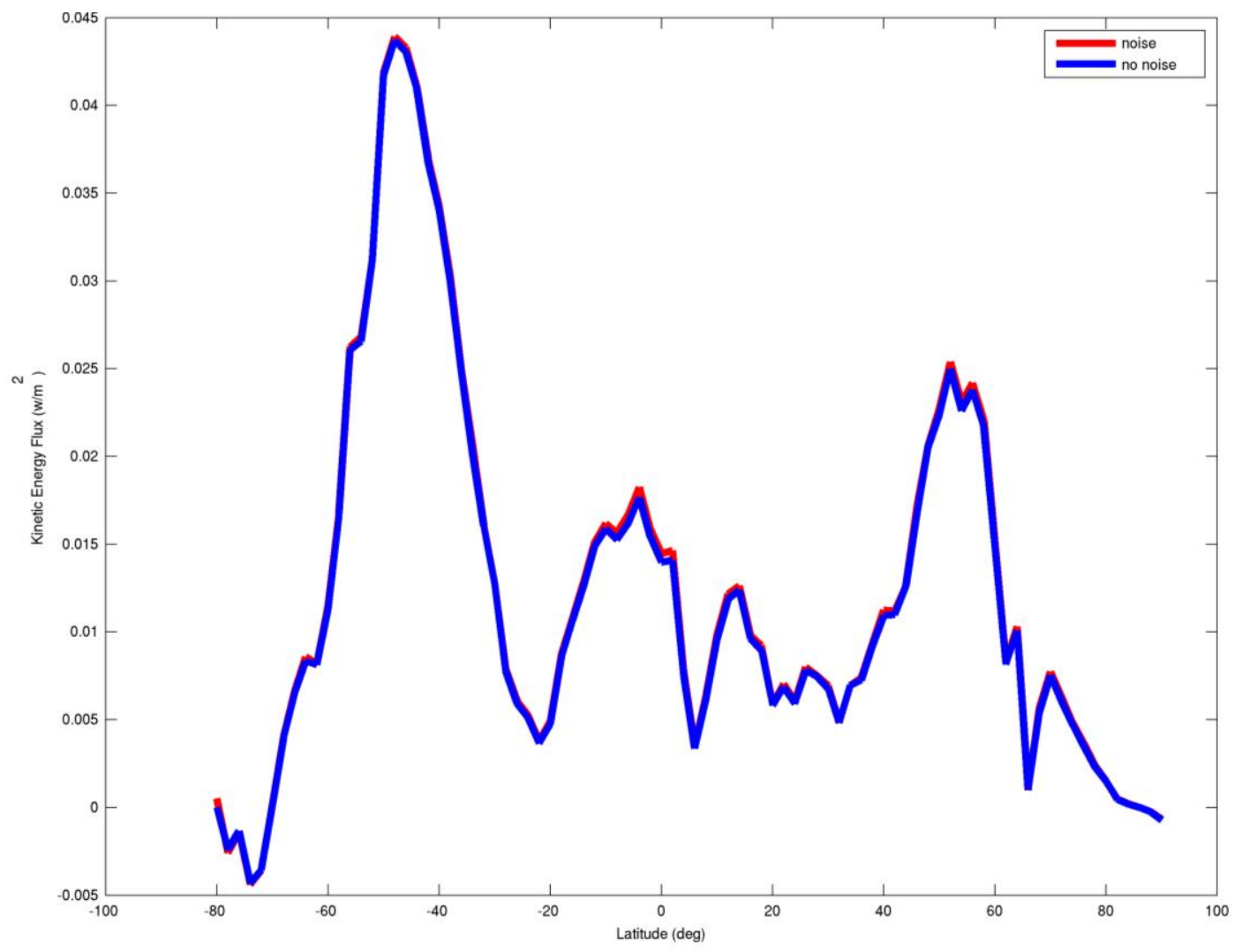

Figure 37: Zonal average comparison of KE flux for noisy and no noise DopplerScat simulations. 


\subsection{Overall Results}

Quite a few different findings have been presented here in quick succession, so this section will attempt to consisely summarize the results. Averaging period in the section is set at 7 days to help match and compare to previous results.

Using the high resolution, $1 / 48^{\circ}$ model, total global wind work is estimated at about 3.22 TW for a 7 day average. Condering Von Storch found a total wind work of about 3.8 TW and used a shorter averaging period, this is in line with previous literature. Comparing this $3.22 \mathrm{TW}$ of total wind power to the combination of geostrophic and Ekman currents, there is again good agreement. Estimating the total currents as the combination of geostrophic and Ekman currents overestimates wind work by just 5 percent. The seven day geostrophic average comes in at about $.32 \mathrm{TW}$ of global wind work. This is about 20 percent lower than the Zhai estimate of $.43 \mathrm{TW}$, probably due to the different models used, different time spans, and different wind stress models. Even still, considering the range of geostrophic wind work estimates $(.4-1.3 \mathrm{TW})$ that have been made in the past, this new geostrophic estimate is on par with the expected results. Ekman currents produce around 3.1 TW of wind work, reasonably in line with the 2.3 TW estimated by Wang and Huang. This difference can again be attributed to different choices of filtering schema. Comparing Ekman wind work to the difference of total and geostrophic work (total-geostrophic), the answers are quite well in line - 3.1 vs. 2.9 TW. This is essentially the same result as comparting total with geostrophic plus Ekman currents. DopplerScat results show very good agreement in total wind work between the noisy observations and the "true" model wind work. Only a 1.5 percent difference is observed between the two on a 8 day average. 
Considering the time sensitivity of these results, the $1 / 24^{\circ}$ model wind work showed a significant increase in total and geostrophic wind work during the Northern Hemisphere summer compared to the winter months. Ekman wind work did not show any difference between seasons. One of the most interesting an useful results of this research is the difference in wind work depending on averaging period. Total and Ekman currents show a 50 percent reduction in wind work when moving from 1 day to 15 day current averages - a result of decorrelation between wind and surface currents. Geostrophic currents displayed the reverse, with a significant increase in wind work as averaging period is increased.

Table 4: Wind work results for each current type and averaging period. Units in TW.

\begin{tabular}{|c|c|c|c|c|c|c|}
\hline Current & Model & 1 day & 3 day & 7 day & 11 day & 15 day \\
\hline Total & $1 / 24^{\circ}$ & 5.53 & 4.10 & 3.23 & 2.93 & 2.78 \\
\hline & $1 / 48^{\circ}$ & 5.46 & 4.11 & 3.22 & 2.86 & 2.65 \\
\hline Geo+Ekman & $1 / 24^{\circ}$ & - & - & 3.43 & - & - \\
\hline & $1 / 48^{\circ}$ & 6.31 & 4.50 & 3.40 & 2.98 & 2.68 \\
\hline Geo & $1 / 24^{\circ}$ & - & - & .34 & - & - \\
\hline & $1 / 48^{\circ}$ & .03 & .18 & .32 & .37 & .40 \\
\hline Ekman & $1 / 24^{\circ}$ & 6.21 & 4.31 & 3.09 & 2.67 & 2.46 \\
\hline & $1 / 48^{\circ}$ & 6.27 & 4.31 & 3.05 & 2.60 & 2.28 \\
\hline Tot-Geo & $1 / 24^{\circ}$ & - & - & 2.89 & - & - \\
\hline & $1 / 48^{\circ}$ & 5.43 & 3.93 & 2.90 & 2.49 & 2.25 \\
\hline DopplerScat & No Noise & & & \multicolumn{2}{|c|}{3.60} & \\
\hline (8 day avgs) & Noisy & & & \multicolumn{2}{|c|}{3.66} & \\
\hline
\end{tabular}




\section{CHAPTER 5 CONCLUSION}

The wind work in the global oceans is estimated with a number of decompositions, with results finding about $3.2 \mathrm{TW}, .32 \mathrm{TW}$, and $3.05 \mathrm{TW}$ for total, geostrophic, and Ekman wind work respectively, when considering a 7 day average. Looking at the closer to instantaneous, 1 day averages, wind work drastically changes to 5.5 TW, .03 TW, and 6.3 TW for total, geostrophic, and Ekman wind works. Averaging period for currents significantly affects the resulting calculated wind work, with greater than 50 percent difference between 1 and 15 days of averaging. Seasonally, wind work appears to be at a maximum during the Northern Hemisphere summer, and at a minimum during the winter months. DopplerScat simulations find that a satellite capable of measuring coincident surface vector winds and surface currents could estimate global wind work to within 2 percent accuracy on an 8 day average with daily snapshots.

There is much still to do in the field of wind work. This research did not consider the work done on inertial oscillations, an important (but smaller than Ekman) part of ageostrophic wind work. The LLC-4320 data set is an extremely powerful tool, and the hourly output could potentially allow for the near instantaneous computation of wind work. This hourly wind work computation is the next logical step, but presents extreme challenges due to the massive data set it would produce. Finally, optimizing vertical viscosity globally in the Ekman current calculations would lead to a more robust calculation. 


\section{REFERENCES}

Duhaut, T. H. A. and D. N. Straub, 2006: Wind stress dependence on ocean surface velocity: implications for mechanical energy input to ocean circulation. J. Phys.

Faller, A. J., 1968: Sources of energy for the ocean circulation and a theory of the mixed layer. Proc. Fifth U.S. Congress of Applied Mechanics, Minneapolis, Minnesota, ASME, 651-672.36, 202-211.

Ferrari, R. and C. Wunsch, 2009: Ocean circulation kinetic energy: Reservoirs, sources, and sinks. Ann. Rev. Fluid Mech., 41, 253-282.

Forget, G., J. Campin, P. Heimbach, and C. Hill. "ECCO Version 4: An Integrated Framework for Non-linear Inverse Modeling and Global Ocean State Estimation." Geoscientific Model Development (2015): n. pag. Web. 28 July 2016.

Munk, W., and C. Wunsch, 1998: Abyssal recipes II: energetics of tidal and wind mixing. Deep Sea Res., 45(12), 1977-2010.

Oort, A. H., L. A. Anderson, and J. P. Peixoto, 1994: Estimates of the energy cycle of the oceans. J. Geophys. Res., 99, 7665-7688.

Scott, Robert, and Yongsheng Xu. "An Update to the Wind Power Input to the Surface Geostrophic Flow of the World Ocean." Deep-Sea Research (2007): n. pag. Web.

Wang, W., and R. X. Huang, 2004: Wind energy input to the Ekman layer. J. Phys. Oceanogr., 34(5), 1267-1275.

Wunsch, C., 1998: The work done by the wind on the oceanic general circulation. J. Phys. Oceanogr., 28(11), 2332-2340. 
von Storch, J.-S., H. Sasaki, and J. Marotzke, 2007: Wind-generated power input to the deep ocean: An estimate using a (1)/(10)degrees general circulation model. J. Phys. Oceanogr., 37(3), 657-672.

Zhai, X., H. L. Johnson, D. P. Marshall, and C. Wunsch, 2012: On the wind power input to the ocean general circulation. J. Phys. Oceanogr., 42, 1357-1365.

Zhai, X., 2013: On the wind mechanical forcing of the ocean general circulation. $J$. Geophys. Res., 118, 6561-6577. 